\title{
Langmuir's Theory of Adsorption: A Centennial Review
}

\section{Authors: Hans Swenson and Nicolas P. Stadie}

This document is the unedited author's version of a Submitted Work that was subsequently accepted for publication in Langmuir copyright (C) American Chemical Society after peer review. To access the final edited and published work, see DOI:10.1021/acs.langmuir.9b00154.

Swenson, Hans, and Nicholas P. Stadie. "Langmuir's Theory of Adsorption: A Centennial Review." Langmuir: the ACS Journal of Surfaces and Colloids 35, no. 16 (April 2019): 5409-5426. DOI:10.1021/acs.langmuir.9b00154.

Made available through Montana State University's ScholarWorks 


\title{
Langmuir's Theory of Adsorption: a Centennial Review
}

\author{
Hans Swenson and Nicholas P. Stadie* \\ Department of Chemistry \& Biochemistry, Montana State University, Bozeman, MT, \\ 59717, USA \\ *E-mail: nicholas.stadie@montana.edu
}

The $100^{\text {th }}$ anniversary of Langmuir's theory of adsorption is a significant landmark for the physical chemistry and chemical engineering communities. Despite its simplicity, the Langmuir adsorption model captures the key physics of molecular interactions at interfaces and laid the foundation for further progress in understanding interfacial phenomena, developing new adsorbent materials, and designing engineering processes. The Langmuir model has had an exceptional impact on diverse fields within the chemical sciences (ranging from chemical biology to materials science), an impact that became clearer with the development of modified adsorption theories and continues to be relevant today. 


\section{Introduction}

Irving Langmuir is known for wide-ranging contributions in chemistry and physics, both in fundamental and applied concepts, and is indelibly linked to the birth of the science of interfaces. His three seminal works on adsorption theory, the foundation for the modern understanding of all types of chemical interfaces, were published in 1916, $1917,{ }^{2}$ and $1918,{ }^{3}$ the last of which is now one century old and the subject of celebration in this historical article. The "superficial" nature of this field of research was playfully acknowledged in the presentation speech of the Nobel Prize in Chemistry to Langmuir in 1932. It was also acknowledged in Söderbaum's very next breath that "in reality, surface chemistry has contributed greatly to the deepening of our knowledge of matter." 4 This remains true today, with major worldwide research efforts, e.g., in heterogeneous catalysis, devoted to phenomena at interfaces.

The history and impact of Langmuir's three seminal works, referred to as Langmuir 1916, Langmuir 1917, and Langmuir 1918, are briefly reviewed herein. The context in which adsorption theory was birthed will first be summarized, followed by a brief account of Langmuir's early work that led directly to the development of his monolayer theory. In addition to the simple, ideal picture of homogeneous monolayer adsorption that has come to be known colloquially as the Langmuir adsorption model, Langmuir also developed several more complex models such as for multilayer and heterogeneous adsorption already in 1918. The ingenious early experiments carried out by Langmuir and his assistant, Mr. S. P. Sweetser, laid the groundwork for future demonstrations of the widespread applicability of this simple theory of site-specific molecular adsorption. Langmuir also emphasized the non-universal applicability of any one equation or model (including, of course, his own monolayer adsorption theory), ${ }^{3}$ and the need to account for a wide breadth of different adsorption mechanisms at play on complicated, real-world surfaces. Hence, the lasting reputation of Langmuir's adsorption theory is both that of a powerful, simple model with far-reaching applicability and textbook elegance, and simultaneously that of a model with few strictly adhering demonstrative systems in the laboratory.

In this review, a statistical mechanical treatment of all six of Langmuir's original cases of adsorption is given, followed by selected contemporary examples of gas-solid adsorbate-adsorbent systems that exhibit representative behavior. Reference to Langmuir's work has risen sharply since 1999 when metal-organic frameworks (MOFs) were revolutionized to include materials with large inner surfaces of crystalline structure; ${ }^{5}$ hence, emphasis is placed herein on examples of Langmuir adsorption on the porous surfaces of MOFs. This extension of Langmuir's "external surface" theory to gas adsorption on the "internal surfaces" of porous materials is one of many natural extensions of Langmuir's original theory to interfaces of all kinds, such as in gas-liquid and liquid-liquid systems, molecular-protein binding, and even in macromolecule or nanocrystal binding. We seek to put Langmuir's now century-old theory into modern context, with a strong emphasis on the firstprinciples simplicity of these concepts and their (cautious) applicability in wide-ranging adsorption systems.

\section{Historical Context}

Early Adsorption Theory. The phenomenon of adsorption has been known since antiquity; ${ }^{6}$ quantitative investigations began in the late $18^{\text {th }}$ century (the earliest known investigations by Scheele, Priestley, and Fontana in 1773-1777, as reviewed more comprehensively elsewhere ${ }^{7-8}$ ), and the term "adsorption" as distinct from "absorption" was originally coined by Emil du Bois- 
Reymond (via Heinrich Kayser ${ }^{9}$ ) in 1881. Adsorption, strictly defined, is the increase in density of a fluid adsorbate at a phase boundary or surface. It remains distinguished from absorption by its limitation to the surface or interface of the sorbent; upon diffusion beyond the interface into the bulk of the sorbent, the adsorbate becomes the absorbate. The term "sorption" was introduced as referring to the ambiguous case encompassing both adsorption and absorption by James W. McBain in 1909.10 This terminology, in principle, remains the convention today.

The history of adsorption theory is intimately tied to both that of intermolecular interactions and the atomic structure of solid-state matter, subjects that were still premature at the turn of the $20^{\text {th }}$ century. It is telling that the forces of attraction between two phases at their interface were still referred to by Langmuir in 1918 as "action at a distance," in quotation marks. ${ }^{3}$ Studies of adsorption were primarily an experimental (as opposed to theoretical) endeavor before 1900. While J. Willard Gibbs developed the earliest thermodynamic theory of "surfaces of discontinuity" in the second part of his landmark work On the equilibrium of heterogeneous substances in 1876, ${ }^{11}$ he specifically remarked as to the quantity of molecules adsorbed being "too small in general to admit of direct measurement." Nevertheless, the terms "surface excess" and "excess quantity of adsorption" were coined by Gibbs and the concept remains of great importance to adsorption theory. The first liquid solute-solid adsorption equilibria were reported by Friedrich Stohmann and Wilhelm Henneberg in $1858^{12}$ (ammonium adsorption on soil) and the first gas-solid adsorption equilibria were reported independently by Chappuis in $1879^{13}$ and Kayser and Joulin in $1881^{9,14}$ (small molecular gases on charcoal), where the delay for gas-solid experiments was due to the more complex apparatus required. Heats of adsorption could be measured as early as 1854 by Favre ${ }^{15-16}$ and practical adsorption applications such as gas separations were well-established by the turn of the century.

Prior to Langmuir's monolayer-based theory first reported in 1916, adsorption was understood such that the density of a fluid at an interface decreases gradually toward the bulk. The theory of adsorption was first focused on the solid surface itself by Herbert Freundlich in 190717, whose empirical equation for adsorption demonstrates respectable utility across relatively wide temperature and pressure ranges still today. However, it is well-known that Freundlich's equation is neither physically founded nor thermodynamically consistent, preventing its fundamental justification. ${ }^{18-19}$ Michael Polanyi alluded to the concept of a characteristic adsorption curve in $1914,{ }^{20}$ and further laid the foundation of what has become known as Polanyi's "potential theory" in 1916. ${ }^{21}$ In a twist of fate, this non-localized theory was rejected at the time for being incongruent with the emerging theories of intermolecular interactions based on electrical forces; nevertheless, it was later developed (together with Fritz London ${ }^{22}$ ) into a widely accepted parallel theory to that of Langmuir's. ${ }^{23}$

Langmuir's theory, on the contrary, was founded on a fundamentally discretized molecular perspective, considering the atomic structure of the surface to be central to a successful description of adsorption. He was strongly influenced by the emerging picture of crystalline solid matter painted by William H. Bragg and his son W. Lawrence Bragg, especially after hearing several lectures given by the elder Bragg during his visit to the eastern United States around the time of the outbreak of World War I (1914-1915). In Langmuir's words: "the importance of the work of W. H. Bragg and W. L. Bragg in its bearing on chemistry has not, as yet, been generally recognized."1 The discrete nature (i.e., sequential filling) of well-defined adsorbed surface layers was the insight first developed by Langmuir and described extensively in Langmuir 1916, with clear reference to the definite, discretized atomic structure of crystals discovered by the Braggs. 
Langmuir pictured monolayer formation to occur based on a specific binding interaction between adsorbate molecules and distinct solid surface sites. This picture was originally founded on the results of experiments performed with hydrogen and other small molecules in the presence of heated metal filaments at very low pressures. In Langmuir 1918, however, it was already broadly generalized to include cases where monolayer or multilayer formation occurs, as well as cases ranging from highly regular surfaces to completely amorphous surfaces containing an abundance of different adsorption sites. The six fundamental cases of adsorption (all designed for adsorption at bulk surfaces) identified by Langmuir laid the foundation for surface science, which has since been adorned with modifications and new theories designed to describe complex, real-word adsorption phenomena, including within molecularly porous materials. ${ }^{6}$

Irving Langmuir. Irving Langmuir, born on January 31st, 1881, received his primary and secondary education in Brooklyn, Paris, and Philadelphia, following his father's work-related movements (as an insurance executive). After the death of his father in 1899, Langmuir entered The School of Mines at Columbia University to study metallurgical engineering, because "the course was strong in chemistry, it had more physics than the chemical course, and more mathematics than the course in physics - and I wanted all three." 24 After receiving his degree, Langmuir, like many American scientists at that time, set off to Germany to obtain a PhD. At the University of Göttingen, his desire to couple scientific research with practical and industrial applications would be honed and affirmed by several great professors, most notably Felix Klein and Walther Nernst. Nernst, who served as Langmuir's primary advisor (despite Friedrich Dolezalek being named as such ${ }^{25}$ ), directed his thesis project of examining the dissociative action of various gases on the surface of a hot platinum filament. ${ }^{26}$ These early experiments would lay the foundation for his lifelong studies of adsorption phenomena.

In early 1906, Langmuir accepted a faculty position at Stevens Institute of Technology in New Jersey, hoping to pursue an academic career in research. After three years of intensive, timeconsuming teaching that afforded little time for research, Langmuir left this position and joined the staff of the General Electric research laboratory, reuniting with an old colleague from Columbia, Colin G. Fink. Langmuir first secured a research position for the summer of 1909, and impressed his coworkers enough to gain a permanent position at the research lab, a position he would hold until his retirement in 1950. At General Electric, Langmuir's independence was consistently protected by the laboratory's director, Willis R. Whitney. His first projects were largely a continuation of his thesis work investigating chemical reactions in the presence of heated metal filaments within glass bulbs, especially tungsten in the presence of hydrogen and other gases. The reaction rates could be slowed and effectively deconvoluted when performed under high-vacuum conditions (a technical achievement of Langmuir's work), culminating in the proposal of a fundamentally new mechanism of heterogeneous chemical reactions. ${ }^{27}$ The two key features of this work, namely the low pressure and inherent role of the metal surface, directly advanced Langmuir toward the breakthrough theory of adsorption based on the concept of molecular layers. Therefore, despite testing his newly found theory on cases of pure physisorption in Langmuir 1918, the original collection of work on which the theory was developed consisted of over a decade of experiments observing the behavior of gases in the presence of heated metal surfaces: pure chemisorption. It is perhaps unsurprising that the monomolecular layer that Langmuir envisioned remains much more accurate in describing the phenomena of chemical adsorption (where the binding strength is strong relative to intermolecular interactions in the bulk gas). 


\section{Langmuir's Theory of Adsorption}

A Kinetic Model. Langmuir originally described gas adsorption at a solid interface as bearing mechanistic similarity to gas-phase condensation at a liquid surface, where inelastic collisions lead to a "lag" or residence time at the location of incidence.1, 28 That is, as gas molecules strike the surface, they are held near the surface by attractive intermolecular forces (akin to condensation) for some short but finite time until leaving into the bulk gas phase again (akin to evaporation). Within this purely kinetic view of adsorption (as shown in Figure 1), and with a newly devised concept of the discretized, surface-oriented nature of adsorption in hand, a simple relationship between equilibrium site occupancy, $\theta$, and the pressure of the gas phase, $P$, was derived. Langmuir made three fundamental assumptions:

(i) the rate of incidence of the molecules in a bulk gas phase on a unit area of adsorbent surface, $r_{i n c}$, is proportional to the pressure at constant temperature via the kinetic theory of gases (adapted from his previous work on saturated metal vapors above their respective solids ${ }^{29}$ ),

(ii) the rate of adsorption (as opposed to metal vapor condensation), $r_{a d s}$, depends not only on the rate of incidence of molecules on the surface, $r_{i n c}$, but also on the probability of adsorption (as opposed to elastic reflection), $p_{a d s}$, and the probability of incidence at a vacant adsorption site (as opposed to at an occupied site), $p_{v a c}$, and

(iii) the rate of desorption is equal to the rate of desorption at maximum surface coverage, $r_{d e s, s a t}$, multiplied by the fractional occupancy of the surface sites by adsorbed molecules, $\theta$ (thereby neglecting any role of adsorbate-adsorbate interactions).

These three assumptions can also be summarized by stating that both the gas phase and adsorbed phase behave ideally (there is no notion of intermolecular interactions) and that every binding site is identical. Combined, they yield the following expression for the equilibrium fractional occupancy of adsorption sites under a gas phase at pressure $P$ :

$$
\theta=\frac{r_{i n c} \cdot p_{a d s}}{r_{d e s, s a t}+r_{i n c} \cdot p_{a d s}}=\frac{K P}{1+K P}
$$

\section{Equation 1}

The Langmuir constant, $K$, is independent of pressure and hence only depends on temperature. When the temperature is invariant, the isotherm can be measured and $K$ experimentally determined, which from this kinetic model is defined as:

$$
K=\frac{1}{\sqrt{2 \pi m R T}} \cdot \frac{p_{a d s}}{r_{\text {des,sat }}}
$$

Equation 2

The Langmuir constant has units of inverse pressure (or inverse concentration for solute adsorption). It is useful to recognize that the surface is precisely half-occupied at the pressure corresponding to $K^{-1}$, as shown in Figure 1, and so represents a "characteristic inverse pressure of adsorption." A more thorough kinetic derivation as originally presented in Langmuir 1918 is reproduced in the Supporting Information using updated nomenclature. 
a

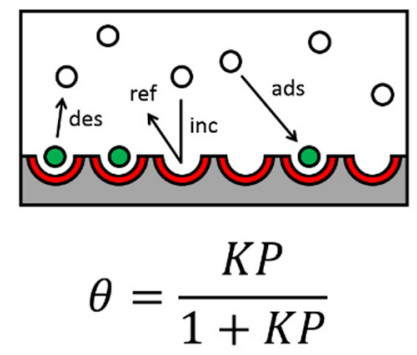

b

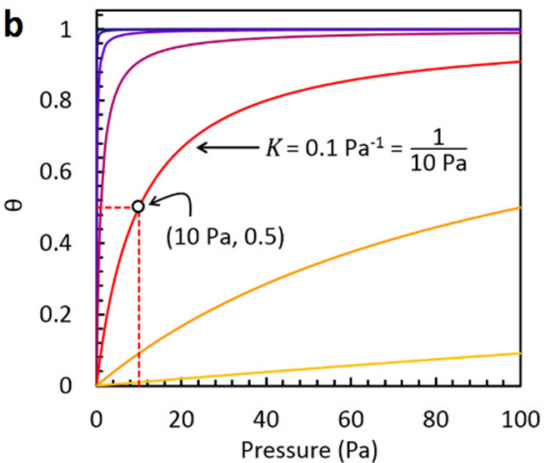

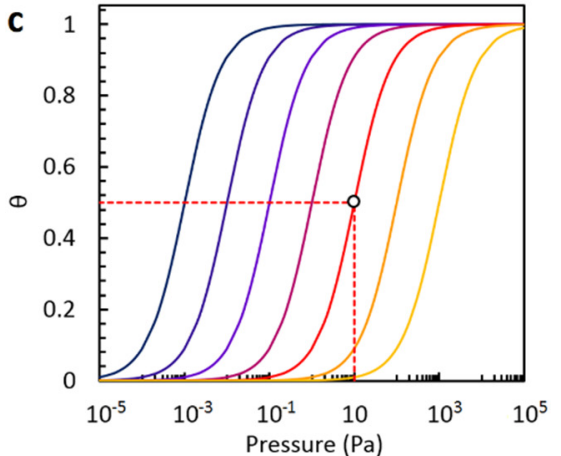

Figure 1. (a) Langmuir's kinetic model of adsorption acknowledges four processes occurring between a gas in equilibrium with a surface (consisting of discretized adsorption sites): incidence (inc), reflection (ref), adsorption (ads), and desorption (des). Treating these fundamental processes with three simple approximations leads to the simple Langmuir isotherm equation. (b-c) The dependence of the Langmuir isotherm on the Langmuir binding constant, $K$, showing hyperbolic shape in the simple plot of fractional adsorption site occupancy, $\theta$, as a function of pressure (b) and sigmoidal shape in the equivalent log-normal plot (c). The magnitude of the binding constant is indicated by color, increasing from $0.001-1000 \mathrm{~Pa}^{-1}$. The characteristic pressure regime corresponding to significant adsorption uptake occurs around $K^{-1}$, as shown for the case of $K=0.1 \mathrm{~Pa}^{-1}$ (red dashed line).

Experimental Results. The original experiments that inspired Langmuir's monolayer hypothesis were investigations of the behavior of hydrogen and oxygen at vacuum pressures inside a glass lightbulb containing a heated tungsten filament, such as that reported in $1912 .{ }^{30}$ Langmuir first observed that the pressure of hydrogen decreased upon exposure to the heated filament over time (especially platinum or palladium). Langmuir concluded that the hydrogen dissociated on the surface of the heated metal, then escaped as a monatomic gas, and finally chemisorbed as atomic hydrogen on the inner glass wall of the bulb. The adsorption of atomic hydrogen on glass was found to be irreversible, although quantitative results as to the amount adsorbed were not reported at that time.

Langmuir continued his experiments with other gases (e.g., $\mathrm{CH}_{4}, \mathrm{NH}_{3}, \mathrm{PH}_{3}, \mathrm{CO}$, and $\mathrm{CO}_{2}$ ) adsorbed on heated metal surfaces and first outlined his monolayer hypothesis in $1916 .{ }^{1}$ Still, equilibrium adsorption isotherm measurements were not presented in Langmuir 1916 or elsewhere at that time. In Langmuir 1917, the gas-solid adsorption interface was temporarily abandoned in favor of the liquid-solid interface. The development of monolayer assemblies at aqueous and other interfaces is not within the focus of the present review, and the interested reader is referred elsewhere. ${ }^{31-32}$

Finally, in Langmuir 1918, methodological studies of the adsorption of numerous gases on three model material surfaces were reported: glass (amorphous silica), muscovite (mica), and platinum. Langmuir performed the experiments along the desorption branch, permitting direct observation of the interplay between physisorption and chemisorption phenomena (the chemisorbed quantity would not be desorbed at low temperature). These were the first set of quantitative adsorption measurements of small molecular gases on solid surfaces of well-known chemistry and geometry. His experiments were performed at extremely low pressure for the time, requiring great care and newly developed vacuum equipment (including a McLeod gauge and Langmuir's invention, the "condensation pump"33, now known as a diffusion pump ${ }^{34}$ ). 

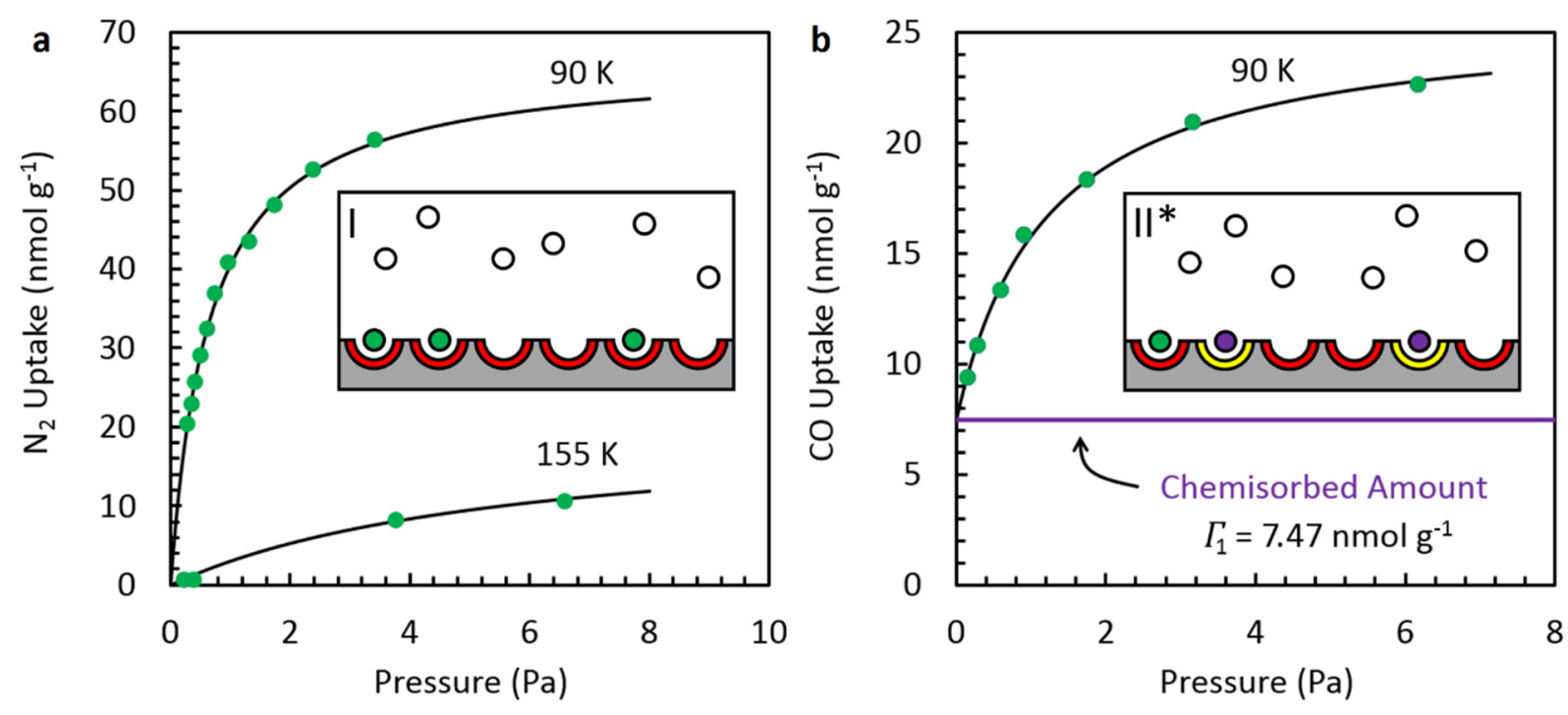

Figure 2. (a) Equilibrium desorption measurements of $\mathrm{N}_{2}$ on mica at 90 and $155 \mathrm{~K}$ as reported in Langmuir 1918, showing single-site Langmuir (SSL) adsorption character. (b) Equilibrium desorption measurements of CO on glass at $90 \mathrm{~K}$ as reported in Langmuir 1918, showing dual-site Langmuir (DSL) adsorption character (the strongly bound species attributed to chemisorption is shown as a solid purple line).

The first set of experiments reported in 1918 were performed on a $24.3 \mathrm{~g}$ sample of muscovite (mica), having an actual (hand-measured) surface area of $0.5750 \mathrm{~m}^{2}$ (specific surface area: 0.0237 $\mathrm{m}^{2} \mathrm{~g}^{-1}$ ). Langmuir confirmed that $\mathrm{N}_{2}$ adsorption on mica between $90-155 \mathrm{~K}$ was purely physical in nature (i.e., physisorption) and could be well-described by his equilibrium kinetic monolayer model (see Figure 2). Modern fitting analysis of these data validate this conclusion, and the results can be used to determine the surface area occupied by a single molecule of $\mathrm{N}_{2}$ in a complete monolayer: $1.69 \mathrm{~nm}^{2}$. This value is significantly larger (by a factor of $\sim 10$ ) than the currently accepted conventional value for $\mathrm{N}_{2}$ adsorption at $77 \mathrm{~K}\left(0.162 \mathrm{~nm}^{2}\right)$; this result may be due to the elevated temperature of Langmuir's 1918 experiments (>90 K). Langmuir indeed recognized that the monolayer densities reported in 1918 were roughly of the correct order of magnitude, but were lower than expected based on a monolayer of liquid by a factor of up to $\sim 9$, even at $90 \mathrm{~K}$. Nevertheless, this evidence strongly favored Langmuir's "monolayer" picture of adsorption since the number of adsorbed $\mathrm{N}_{2}$ molecules did not exceed that of a liquid monolayer but approached a well-defined maximum.

Langmuir's second set of experiments were performed on nearly 200 individual glass microscope slides, each defected slightly to prevent stacking and permit free access of the gas to the amorphous surface. The total sample mass was $\sim 36.7 \mathrm{~g}$ (based on a reported volume of $13.6 \mathrm{~mL}$ ), and the total surface area was measured to be $0.1966 \mathrm{~m}^{2}$ (specific surface area: $0.00535 \mathrm{~m}^{2} \mathrm{~g}^{-1}$ ). Several parts of the apparatus used in the preceding mica experiments were replaced to permit higher accuracy adsorption measurements necessitated by the extremely low binding strength of the glass surface. Impressively, total quantities of adsorbed gas as minute as $20 \mathrm{nmol}$ on the entire sample (the lowest amount detected is the last point of Langmuir's argon desorption isotherm, at $0.1 \mathrm{~Pa}$ and 90 $\mathrm{K})$ could be detected. It is unclear which attribute of the glass surface that Langmuir set out to contrast with the muscovite: the amorphous nature of the glass surface (Case III, described below, is theoretically developed for amorphous surfaces with infinitely many different sites) or merely its 
different chemistry (as $\mathrm{SiO}_{2}$ ). In any case, the most remarkable results were for $\mathrm{CO}$ adsorption on glass at $90 \mathrm{~K}$; the $\mathrm{CO}$ could not be fully desorbed under vacuum, motivating a multi-site monolayer model (Case II) where one type of site is characterized by weak adsorption (physisorption) and another by strong adsorption (chemical adsorption, or chemisorption), as shown in Figure 2.

Langmuir's third set of experiments were performed to differentiate the nature of chemical and physical adsorption on platinum metal foil, and to unravel some of the complexities of the platinumcatalyzed oxidation of $\mathrm{H}_{2}$ and $\mathrm{CO}$. A small square of $0.010 \mathrm{~mm}$ thick foil was used, washed and carefully folded, having a total mass of $4.03 \mathrm{~g}$ and a total surface area of $0.0312 \mathrm{~m}^{2}$ (specific surface area: $0.00774 \mathrm{~m}^{2} \mathrm{~g}^{-1}$ ). Langmuir found that at room temperature and below, only very small quantities of $\mathrm{H}_{2}, \mathrm{O}_{2}$, and $\mathrm{CO}$ could be adsorbed on platinum, far below that of the calculated monomolecular layer. A series of experiments at above ambient pressure were then reported, demonstrating several key features of the platinum system, namely: chemical reaction, catalysis, and hysteresis (depending on what is now known as "activation"). The resulting insights into the catalytic reaction mechanisms at play on the platinum surface represented important early contributions to research in the budding field of heterogeneous catalysis, where Langmuir's work would have long-lasting impact. ${ }^{35}$

Corroborating Langmuir's Theory. Numerous investigations were carried out in the decades after 1918 to test Langmuir's theory in other simple gas-solid adsorption systems. It has been found that in the routine characterization of practical materials such as heterogeneous catalysts and porous adsorbents (usually with subcritical adsorptive fluids like $\mathrm{N}_{2}$ ), multilayer adsorption is far more often verified than simple monolayer adsorption. This led to a more detailed theory of multilayer adsorption championed by Stephen Brunauer, Paul Emmett, and Edward Teller ${ }^{36}$ (a specific modification of Langmuir's Case VI referred to as BET theory, described below) that remains wellknown today. Langmuir remained silent during his lifetime on the relative utility of his theory as compared to that of Brunauer, Emmett, and Teller, a fact that was (perhaps rather sensitively) interpreted by Brunauer to imply resentment. ${ }^{37}$ However, it is clear that, at least in the case of gas adsorption on external solid surfaces, no such defense of the relative applicability of each equation need be made. Langmuir's simplest monolayer formulation is usually applicable when the strength of interaction between the adsorbate and solid surface is far stronger than that between two adsorbate molecules, while BET theory (which is itself an extension of Langmuir's own multilayer theory) is applicable when the interactions are more similar. It is merely a question of which regime represents the system of interest. We note that Langmuir's original data (e.g., $\mathrm{N}_{2}$ adsorption on mica and $\mathrm{CO}$ on glass at $90 \mathrm{~K}$ as shown in Figure 2) were not collected at high enough pressure to discern the accumulation of multiple adsorption layers.

Numerous adsorption isotherm equations are now generally used to interpolate and/or model experimental adsorption equilibria, typically based on one of three fundamental underlying approaches: Freundlich's empirical equation, Langmuir's surface-based equations, or Polanyi's potential theory. Surfaces within porous solids, in particular within microporous solids, present as highly complex to accurately model, and typically cannot be adequately addressed with a simple Langmuir-type analysis. For further information, the reader is referred to several more detailed reviews ${ }^{6-7,38}$ of the subject.

Hill's Equilibrium Theory of Protein Binding. An analogous theory to Langmuir's theory of monolayer gas adsorption on solid surfaces had in fact already been independently developed significantly earlier than 1916, specifically to explain the oxygen "dissociation curves" associated 
with hemoglobin in human and animal blood. Like gas adsorption on a finite number of solid surface sites as a function of pressure, the binding of a ligand (e.g., oxygen) to functional sites on a finite number of proteins in solution will reach a value at equilibrium that depends on the concentration of the ligand (the adsorbate). Archibald V. Hill, an undergraduate student at Trinity College in Cambridge, based this theory on data reviewed by Barcroft and Camis in the Journal of Physiology in 1909,39 relating the fraction of oxygen-saturated protein to the partial pressure (tension) of oxygen in various aqueous solutions. It had earlier been observed by Christian Bohr et al. that the shape of such dissociation curves was sigmoidal, indicating cooperative binding. ${ }^{40}$ Hill, who would later go on to receive the Nobel Prize (in Medicine in 1922), suggested that the data could be accurately represented by an equation derived from a mass-action framework for the reversible combination of a ligand (or general binding moiety) with a discrete set of receptor structures (binding sites) at equilibrium. ${ }^{41}$

The same general laws of mass action at equilibrium underpin both the Hill and Langmuir equations, with a key difference in emphasis for Hill's development: the allowance for multiple binding sites (and hence cooperative adsorption) on a single protein. The simpler Hill equation for systems with only a single receptor site per protein is identical to the Langmuir equation for simple, single-site gas adsorption on a solid surface. It can thus be derived using the same equilibrium kinetic model (i.e., the law of mass action for a simple combination "reaction") as given above. A summary of the mass action approach (for either single-site gas adsorption or single-receptor protein binding) can be made as follows:

\section{Single-Site Gas Adsorption}

$$
\begin{gathered}
\mathrm{A}_{(\mathrm{g})}+\text { site } \leftrightarrow \mathrm{A}_{(\mathrm{a})} \\
\theta=\frac{K P}{1+K P}
\end{gathered}
$$

\section{Single-Molecule Receptor Binding}

$$
\begin{array}{cc}
\mathrm{L}_{(\mathrm{aq})}+\mathrm{R}_{(\mathrm{aq})} \leftrightarrow \mathrm{LR}_{(\mathrm{aq})} & \text { Equation 3 } \\
\theta=\frac{K[\mathrm{~L}]}{1+K[\mathrm{~L}]} & \text { Equation 4 }
\end{array}
$$

For oxygen binding to myoglobin, for example, this simple treatment is consistent with experimental results, indicating that a single receptor binds oxygen on each protein since no adsorbate-adsorbate cooperation is detected (see Figure 3).

The more general Hill equation for binding structures with multiple receptor sites per protein (first reported in its present form in $1910^{42}$ ) can be derived using a similar mass-action at equilibrium model, but for a ligand binding "reaction" involving multiple receptors on the same protein:

Multi-Molecule Receptor Binding

$$
\begin{aligned}
n \mathrm{~L}_{(\mathrm{aq})}+\mathrm{R}_{n(\mathrm{aq})} \leftrightarrow \mathrm{L}_{n} \mathrm{R}_{n(\mathrm{aq})} & \text { Equation } 5 \\
\theta=\frac{K[\mathrm{~L}]^{n}}{1+K[\mathrm{~L}]^{n}} & \text { Equation 6 }
\end{aligned}
$$

Hill originally pictured single-receptor proteins that were "coagulated" together, causing interaction between the binding sites on closely held proteins; this has since given way to the modern understanding of multiple ligand receptors on each protein, as determined by X-ray crystallography. ${ }^{43}$ For oxygen binding to hemoglobin, simple ligand-binding theory (corresponding to Equation 4) is not consistent with experimental results, nor were other empirical equations such 
as that proposed by Wolfgang Ostwald (in the vein of Freundlich's empirical equation for gas adsorption). ${ }^{39}$ Instead, Hill showed that experimental results were consistent with either a combination of Equation 4 and Equation 6 (where $n=2$ was imposed) or simply by Equation 6 alone (where $n$ was allowed to vary as an independent parameter). In the latter case, the value of $n$ was initially determined to be $\sim 1.4$, indicating significant cooperation between the oxygen binding sites on a single, tetrameric hemoglobin protein (see Figure 3). Modern experimental data confirm a higher value of $n=\sim 2.6 .^{44}$ This cooperative binding of oxygen between the four ferrous heme complexes in a single hemoglobin protein has crucial implications for oxygen transport and delivery in biological systems. It is notable that Hill's theory did not allow for partial occupation of each protein (e.g., as in imposing individual contributions to the total adsorbed amount from states corresponding to $n=1,2,3,4 \ldots$ ) in favor of a simpler equation with fewer independent parameters.
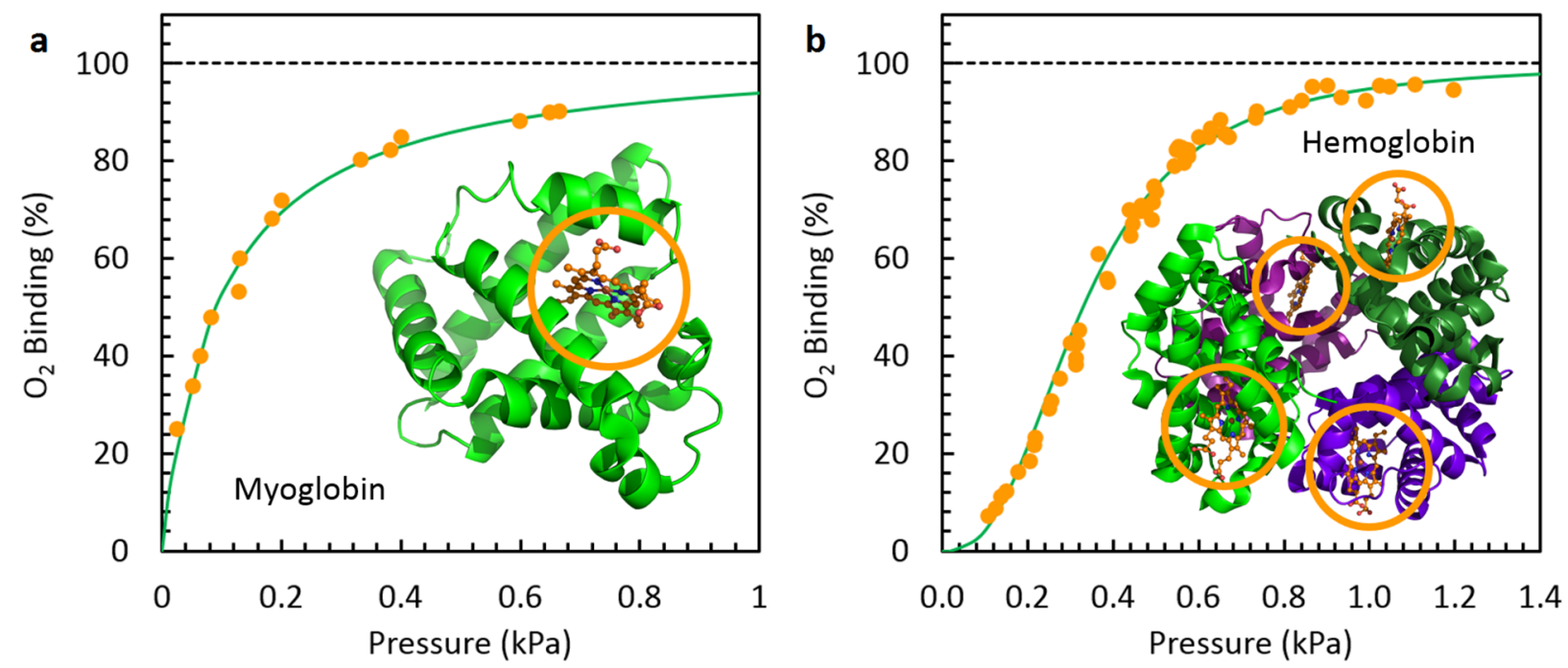

Figure 3. (a) Equilibrium $\mathrm{O}_{2}$ binding on myoglobin, as reported by Rossi-Fanelli and Antonini45, showing single-site Langmuir (SSL) character. (b) Equilibrium $\mathrm{O}_{2}$ binding on hemoglobin, as originally measured by Ferry and Green ${ }^{46}$ and then normalized by Pauling ${ }^{47}$, showing cooperative adsorption (CA) character. Myoglobin (protein structure inset left) has 1 binding site that binds $\mathrm{O}_{2}$, while hemoglobin (protein structure inset right) has 4 binding sites that semi-cooperatively bind $\mathrm{O}_{2}$, lending a Hill coefficient of $\sim 2.6$.

The Hill coefficient, $n$, is recognized as an indicator of cooperative interactions, since it describes the number of molecules bound per receptor (or, in the gas-solid adsorption system, the number of adsorbed molecules per surface site). This was originally posited to be equal to, in the case of protein binding, the integer number of receptors per protein; in reality, however, protein binding requires more complexity to accurately describe, and so the Hill coefficient is only loosely indicative of the cooperativity between receptor sites. Indeed, the value of $n$ is often a non-integer. Langmuir also considered the possibility of cooperative adsorption phenomena (Case IV, detailed below) but did not explicitly work out the equation for adsorption between $n$ neighboring adsorbates in 1918 . The Hill equation continues to play an important role in biological analysis across a wide variety of applications, including drug discovery. ${ }^{48}$ The underlying physical rationale for cooperative effects in ligand binding, including of oxygen on hemoglobin, ${ }^{49}$ remains an important topic of research today.

\section{Langmuir's Classifications of Adsorption}


Langmuir acknowledged that the diversity of surface chemistry and structural geometry exhibited by solid materials, in conjunction with the diversity in size and character of adsorbate species, manifests in widely different physical mechanisms of adsorption. ${ }^{1}$ Langmuir identified and classified six distinct, simple mechanisms of adsorption in Langmuir 1918, and then derived the equation governing the amount adsorbed, $n_{\mathrm{a}}$, as a function of pressure, $P$, at constant temperature, $T$, for each case. These six classifications are depicted schematically in Figure 4. Derivations of each case (including some special sub-cases, indicated by an asterisk) follow, along with examples of representative adsorption behavior reported in the recent literature.

Although Langmuir originally employed equilibrium mass-action theory to derive the adsorption isotherm for each case, a more fundamental statistical mechanical derivation is presented herein. Each begins by prescribing the potential energy field created by the surface of the material, $U(\boldsymbol{x})$, where $x \in R^{3}$ is a point on the solid surface. The potential energy field arises from relevant electrostatic and London dispersion forces between the surface of the material and the adsorbate molecules. Using standard statistical mechanical methods, the adsorption isotherm then follows directly from the potential energy field, $U(\boldsymbol{x})$, and a stipulation for how the adsorbed molecules interact with each other. This is a guest-host perspective where it is assumed that the adsorbate does not affect the structure of the adsorbent.

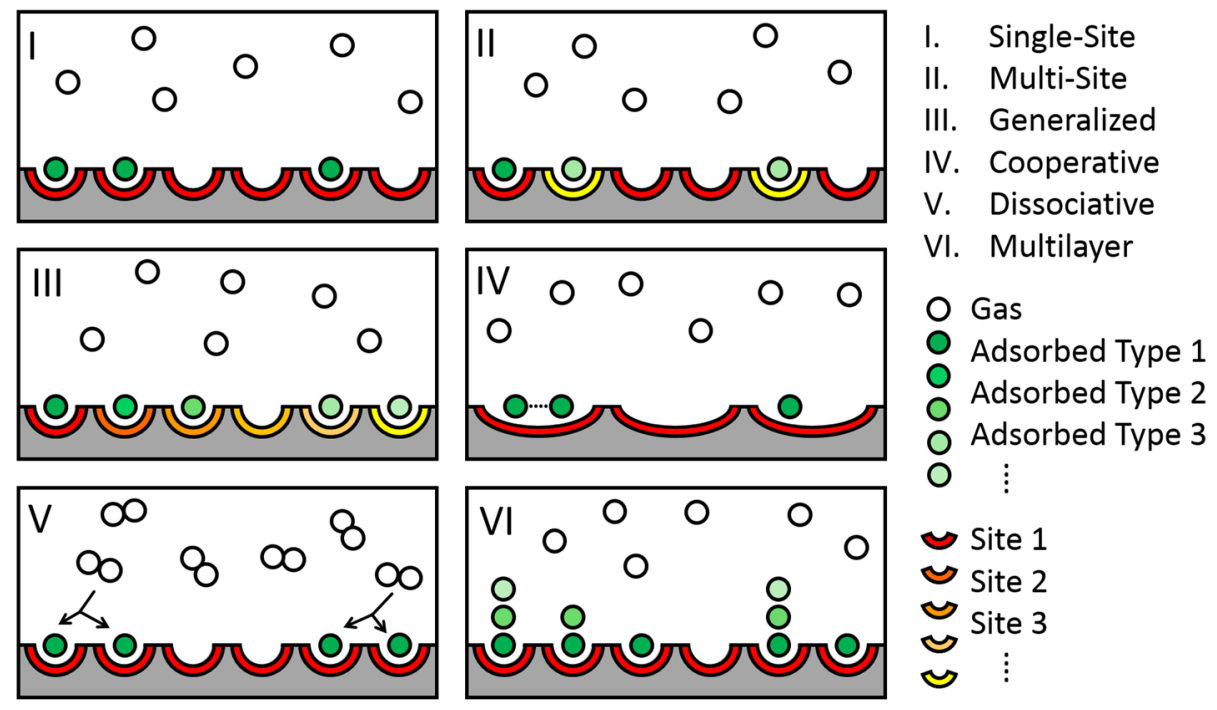

Figure 4. Six adsorption classifications were proposed by Langmuir in 1918: single-site or "simple" (SSL) adsorption, multi-site (MSL) adsorption (of which a sub-case is dual-site (DSL) adsorption), generalized multi-site (GL) adsorption, cooperative adsorption (CA) (of which a sub-case is quadratic adsorption (QA)), dissociative adsorption (DA), and multilayer adsorption (MLA).

\section{Case I: Single-Site (Simple) Langmuir Adsorption (SSL)}

The simplest case of gas-solid adsorption is that on a surface (covering a unit gram of adsorbent material) composed of $\Gamma$ identical elementary adsorption sites, each capable of hosting only a single adsorbed molecule, $\mathrm{A}_{(\mathrm{a})}$. In the simplest case, adsorbate-adsorbate interactions on the surface are neglected, and the bulk gas phase is taken to be ideal. If the potential energy of an adsorbate at any binding site on the surface is uniform and taken to be equal to $U_{1}$ (with dimensions of energy per 
molecule), the adsorption isotherm equation (the adsorbed quantity, $N_{\mathrm{a}}$, as a function of bulk gas pressure, $P$ ) is found to be:

$$
N_{\mathrm{a}}(P)=\Gamma \theta=\Gamma \frac{K_{1} P}{1+K_{1} P}
$$

where $K_{1}$ (having dimensions of inverse pressure) is the Langmuir binding constant equivalent to that defined in Equation 2.

Statistical Mechanics Derivation. We consider a crystalline surface with a unit cell containing a single, molecularly accessible adsorption site. The potential energy of an adsorbate molecule at any point $\boldsymbol{x}$ within a single unit cell is described as:

$$
U(x)= \begin{cases}U_{1} & \text { if } x \in \Omega_{B} \\ \infty & \text { if } x \notin \Omega_{B}\end{cases}
$$

Equation 8

where $\Omega_{B}$ is the set of points comprising the adsorption site (i.e., $B$ is for "binding site") of volume $V_{B}=\left|\Omega_{B}\right|$ (the notation $|x|$ where $x$ is a set of points is used to indicate the "volume of $x$ "). The potential energy is infinity outside the adsorption site. When the binding site is unoccupied, the potential energy of the unit cell is 0 . The total volume of the unit cell, where $\Omega$ is the set of points comprising the unit cell, is then $V=|\Omega|$.

The fundamental assumptions inherent to the SSL model are:

(i) every binding site is identical,

(ii) there are no interactions between adsorbed molecules, and

(iii) there are no interactions in the gas phase (i.e., it is an ideal gas).

The adsorbed phase is taken to have a constant volume and allowed to exchange internal energy and particles with a thermal (at constant $T$ ) and "chemical" (at constant $\mu$ ) reservoir. The relevant statistical mechanical representation is the grand canonical ensemble; at any given site, the grand canonical partition function, $\xi$, is a sum over all (two) possible states of the site:

$$
\xi(\mu, V, T)=1+\Lambda^{-3} e^{\beta \mu} \int_{\Omega} e^{-\beta U(x)} d x=1+\Lambda^{-3} V_{B} e^{-\beta U_{1}} e^{\beta \mu} \quad \text { Equation } 9
$$

where $\beta$ is the inverse temperature $\left(k_{B} T\right)^{-1}, k_{B}$ is the Boltzmann constant, $\Lambda$ is the thermal de Broglie wavelength of the adsorbate molecule, and $\mu$ is the chemical potential of the bulk gas phase in equilibrium with the adsorbed phase. The first and second terms in Equation 9 correspond to the single adsorption site being empty and occupied, respectively. In this configurational partition function, the "state" of the adsorptive molecule is a continuum (and integration is performed), with the thermal de Broglie wavelength accounting for its underlying discrete nature.

The adsorption sites in the SSL model are independent and hence the total grand canonical partition function of the entire adsorbed phase over all $\Gamma$ adsorption sites, $\Xi$, is factorizable:

$$
\Xi(\mu, V, T)=\xi^{\Gamma}
$$

Equation 10

Finally, the expected number of adsorbed molecules, $N_{\mathrm{a}}$, follows from a derivative of the total partition function: 


$$
\left\langle N_{\mathrm{a}}\right\rangle=\left(\frac{\partial \log \Xi}{\partial(\beta \mu)}\right)_{\beta, V}=\Gamma \frac{\Lambda^{-3} V_{B} e^{-\beta U_{1}} e^{\beta \mu}}{1+\Lambda^{-3} V_{B} e^{-\beta U_{1}} e^{\beta \mu}}
$$

Equation 11

At thermodynamic equilibrium, the bulk gas phase (acting as a "chemical reservoir") imposes its chemical potential on the adsorbed phase, ${ }^{50}$ which for an ideal gas is given by:

$$
\mu=k_{B} T \log \left(\Lambda^{3} \beta P\right)
$$

Equation 12

By substituting Equation 12 into Equation 11 to replace chemical potential with the bulk gas pressure, the common representation of the Langmuir adsorption isotherm (Equation 7) is obtained, with the Langmuir constant explicitly defined as:

$$
K_{1}=\beta V_{B} e^{-\beta U_{1}}
$$

Equation 13

This equation relates the Langmuir constant to the temperature, $T$ (via $\beta$ ), the binding site volume, $V_{B}$, which determines the translational entropy of an adsorbed molecule, and to the potential energy of adsorption, $U_{1}$.

Modern Example: Xenon Adsorption on SBMOF-1. The adsorption of xenon on SBMOF-1 at room temperature between $0.1-100 \mathrm{kPa}$ is a representative example of SSL adsorption. ${ }^{51}$ SBMOF-1, also known as $\mathrm{Ca}(\mathrm{sdb})$ ( $\mathrm{sdb}=4,4$-sulfonyldibenzoate), is comprised of one-dimensional pores with constrictions of $\sim 4.2 \AA$ in diameter; in situ single-crystal X-ray diffraction studies and molecular models reveal that xenon adsorbs at well-defined, uniform pockets (binding sites) within these pores. The structure of SBMOF-1 and contours of the potential energy of Xe within its pores (via calculations based on the Universal Force Field) are shown in Figure 5a. Since each well-defined, uniform pocket is capable of holding only a single atom, the experimental Xe adsorption isotherm exhibits clear SSL character. The maximum binding site occupancy, $\Gamma$, obtained by fitting the experimental data to the Langmuir model, is closely matched to the theoretical maximum number of Xe atoms per unit gram of material as estimated by inspecting the crystal structure (shown as a dashed black line in Figure 5a).
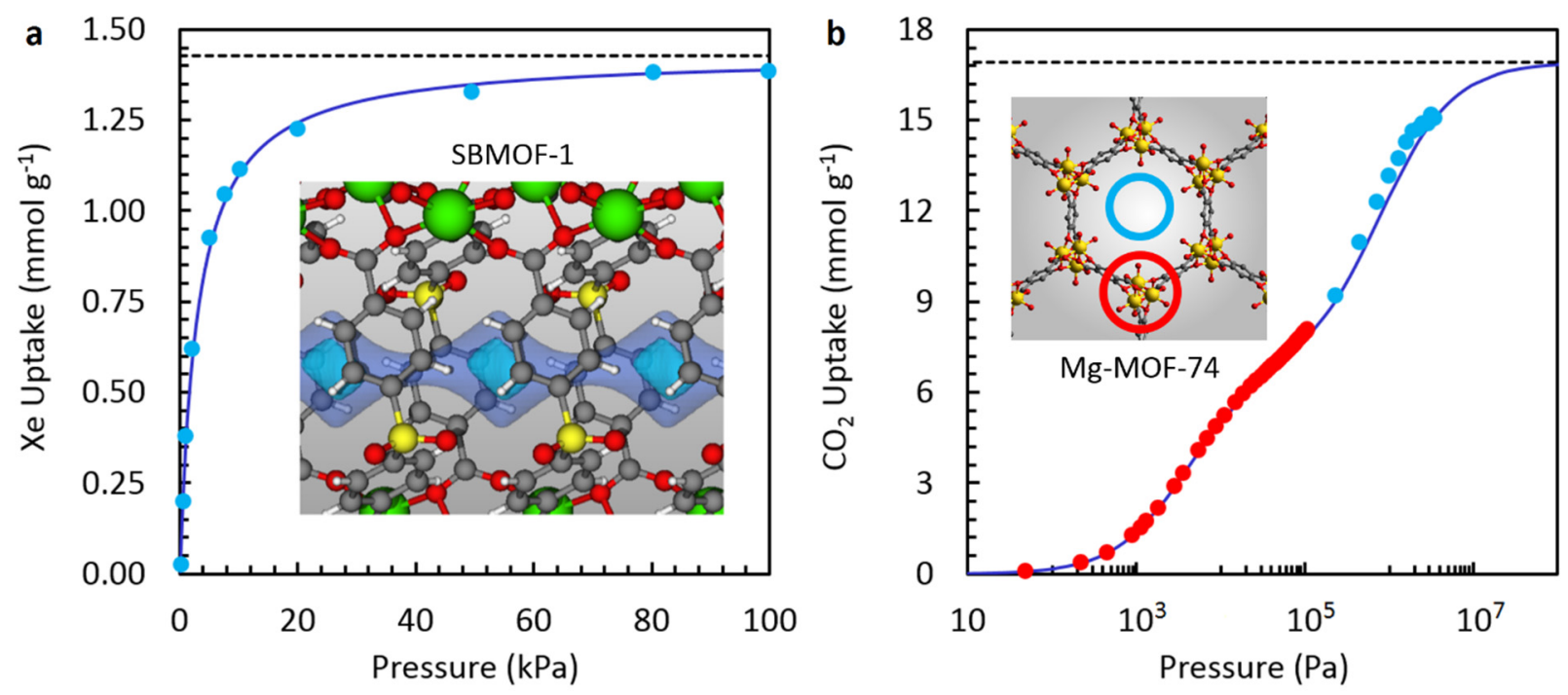

Figure 5. (a) Equilibrium adsorption uptake of Xe on SBMOF-1 at $298 \mathrm{~K}, 51$ an example of single-site Langmuir (SSL) adsorption, and (b) equilibrium adsorption uptake of $\mathrm{CO}_{2}$ on Mg-MOF-74 at 313 $\mathrm{K}, 52$ an example of dual-site Langmuir (DSL) adsorption. 


\section{Case II: Multi-Site Langmuir Adsorption (MSL)}

We now consider a surface that offers more than one type of elementary adsorption site, yet where each site may still only accommodate a single adsorbed molecule, all binding sites are independent, and adsorbate-adsorbate interactions are negligible. The contribution of each type of site to the total amount adsorbed is simply additive, leading to the MSL adsorption isotherm equation:

$$
N_{\mathrm{a}}(P)=\sum_{i=1}^{\text {types }} \Gamma_{i} \frac{K_{i} P}{1+K_{i} P}
$$

Equation 14

where the sum is over the number of different types of binding site, $K_{i}$ is the Langmuir constant for sites of type $i$, and the total number of sites is simply:

$$
\Gamma=\sum_{i=1}^{\text {types }} \Gamma_{i}
$$

Equation 15

\section{Case II*: Dual-Site Langmuir Adsorption (DSL)}

The simplest case of MSL adsorption is that where the surface offers two distinct types of adsorption site, known as the dual-site Langmuir (DSL) model. If the surface is comprised of $\Gamma_{i}$ adsorption sites of site volume $V_{B, i}$ and characteristic binding energy $U_{i}$, for $i \in\{1,2\}$, the adsorption isotherm equation is:

$$
N_{\mathrm{a}}(P)=\Gamma_{1} \frac{K_{1} P}{1+K_{1} P}+\Gamma_{2} \frac{K_{2} P}{1+K_{2} P}
$$

Equation 16

A key illustration of the DSL model is that, as more gas adsorbs, gas molecules tend to fill the adsorption sites on a heterogenous surface that offer the lower potential energy before occupying those with a higher potential energy. The disparity in binding energy between the two sites and the temperature determine the extent to which the more favorable sites are filled first. At dilute conditions, the ratio of the number of adsorption sites occupied (sites of type 1 to those of type 2 ) is:

$$
\frac{N_{\mathrm{a}, 1}}{N_{\mathrm{a}, 2}}=\frac{K_{1} \Gamma_{1}}{K_{2} \Gamma_{2}}=\frac{\Gamma_{1}}{\Gamma_{2}} \frac{V_{B, 1}}{V_{B, 2}} e^{-\beta\left(U_{1}-U_{2}\right)}
$$

Equation 17

following from the derivation presented below. A higher number of adsorption sites, $\Gamma_{i}$, and larger binding site volume, $V_{B, i}$, tend to increase the discrepancy in occupancy between the two types of site. The discrepancy in the potential energy of adsorption, $U_{i}$, has an exponential influence on the differences in occupancy, modulated by the temperature. At higher temperatures, the difference in potential energy of adsorption between the two types of site is less consequential as entropy increases the exploration of both types of site; at lower temperatures, a greater difference in occupancy is observed for a given difference in potential energies.

Statistical Mechanical Derivation. We consider a surface containing two distinct types of adsorption site. Each site provides a spatially homogeneous potential energy of adsorption, $U_{i}$, for a single gas molecule, where $i \in\{1,2\}$. The single-site grand canonical partition function, $\xi_{i}$, for each type of site is the same as that derived for SSL adsorption: 


$$
\xi_{i}(\mu, V, T)=1+\Lambda^{-3} V_{B, i} e^{-\beta U_{i}} e^{\beta \mu}
$$

Equation 18

The total grand canonical partition function is factorizable since each adsorption site is independent, and thus:

$$
\Xi(\mu, V, T)=\xi_{1}^{\Gamma_{1}} \xi_{2}^{\Gamma_{2}}
$$

Equation 19

The expected number of adsorbed gas molecules is then:

$$
\left\langle N_{\mathrm{a}}\right\rangle=\left(\frac{\partial \log \Xi}{\partial(\beta \mu)}\right)_{\beta, V}=\sum_{i=1}^{2} \Gamma_{i} \frac{\Lambda^{-3} V_{B, i} e^{-\beta U_{i}} e^{\beta \mu}}{1+\Lambda^{-3} V_{B, i} e^{-\beta U_{i}} e^{\beta \mu}} \quad \text { Equation } 20
$$

This equation can be rewritten as $\left\langle N_{\mathrm{a}}\right\rangle=\left\langle N_{\mathrm{a}, 1}\right\rangle+\left\langle N_{\mathrm{a}, 2}\right\rangle$, where $\left\langle N_{\mathrm{a}, i}\right\rangle$ is the expected number of molecules adsorbed on the sites of type $i$. Thus, the contribution by each type of site is purely additive. Using Equation 12 to replace chemical potential with the pressure of the bulk gas phase, the DSL adsorption isotherm (Equation 16) is achieved.

Modern Example: $\mathrm{CO}_{2}$ Adsorption on Mg-MOF-74. A representative example of DSL adsorption is that of $\mathrm{CO}_{2}$ on Mg-MOF-74 at $313 \mathrm{~K}$, as shown in Figure $5 b^{52}$ The Mg form of MOF-74, also known as $\mathrm{Mg}_{2}$ (dobdc) (dobdc = 2,5-dioxido-1,4-benzene dicarboxylate), is comprised of one-dimensional, hexagonal channels bounded by exposed square pyramidal $\mathrm{Mg}^{2+}$ cations that are known to be sites of strong adsorption for many small gas molecules. The inflection in the adsorption isotherm at $\sim 10^{5} \mathrm{~Pa}$ is a clear indication of DSL character, and is due to the large disparity in binding energy between the adsorption sites near the exposed $\mathrm{Mg}^{2+}$ metal centers and elsewhere on the surface ( $\sim 42$ and $\sim 24 \mathrm{~kJ} \mathrm{~mol}^{-1}$, respectively). Fitting analysis performed herein yields two distinct Langmuir constants: $K_{1}=2.49 \times 10^{-4} \mathrm{~Pa}^{-1}$ and $K_{2}=1.28 \times 10^{-6} \mathrm{~Pa}^{-1}$. In contrast with CO adsorption on glass as reported in Langmuir 1918, both distinct adsorption sites in Mg-MOF-74 are of physisorptive type, allowing the entire adsorption isotherm to be reversible at $313 \mathrm{~K}$.

\section{Case III: Generalized Langmuir Adsorption (GL)}

While Case II is designed to handle multiple types of distinct adsorption sites, an amorphous material may comprise an intractable number of different adsorption sites with differing affinities toward the adsorbate. Langmuir suggested to treat the distribution of site affinities in such an amorphous material as a continuum. ${ }^{3}$ Neglecting adsorbate-adsorbate attractions, the adsorption isotherm follows from the distribution of binding energies among the adsorption sites.

Let $m(U) d U$ be the number of elementary spaces on the entire surface with a characteristic binding energy between $U$ and $U+d U$. We neglect to employ a unit cell, permitting the treatment of either crystalline or amorphous surfaces. Necessarily, $\int_{-\infty}^{\infty} m(U) d U=\Gamma$, the total number of elementary spaces offered by the surface. When the role of adsorbate-adsorbate attractions can be neglected, the adsorption isotherm is simply the continuum analogy to the MSL model:

$$
N_{\mathrm{a}}(P)=\int_{-\infty}^{\infty} \frac{K(U) P}{1+K(U) P} m(U) d U
$$

Equation 21

where $K(U)=\beta V_{B} e^{-\beta U}$ is the Langmuir constant of a site offering energy of adsorption $U$.

Langmuir noted that an impractically intimate knowledge of the surface structure would be needed to write $m(U)$ explicitly. Nevertheless, Langmuir speculated that conclusions about the character of 
$m(U)$ could in fact be drawn from the experimental adsorption isotherm, ${ }^{3}$ which was later validated by Sips. ${ }^{18} \mathrm{~A}$ strong assumption inherent to Equation 21 is that $V_{B}$ is the same for all adsorption sites, ${ }^{53}$ but Sips noted that $V_{B}$ can, in principle, also be written as a function of $U .{ }^{19} \mathrm{~A}$ simple case of Equation 21 is where the distribution is a Dirac delta function centered at $U_{0}$, or in other words where $m(U)=\Gamma \delta\left(U-U_{0}\right)$. In this case, all adsorption sites are identical and the SSL model is recovered (as in Equation 7).

\section{Case III*: Uniform Langmuir or Unilan Adsorption (UL)}

An especially useful case of Equation 21 is known as the Unilan (for "uniform Langmuir"), or UL model. ${ }^{54}$ In this case, all of the binding sites are unique and the binding energies are taken to be uniformly distributed between $U_{\min } \leq U \leq U_{\max }{ }^{19}$ This is expressed as:

$$
m(U)= \begin{cases}\frac{\Gamma}{U_{\max }-U_{\min }} & \text { if } U \in\left[U_{\min }, U_{\max }\right] \\ 0 & \text { otherwise }\end{cases}
$$

Equation 22

By imposing this distribution, Equation 21 results in the Unilan equation:

$$
N_{\mathrm{a}}(P)=\frac{\Gamma}{2 s} \ln \left(\frac{1+K_{\text {avg }} e^{s} P}{1+K_{\text {avg }} e^{-s} P}\right)
$$

Equation 23

where $s$ is related to the range of adsorption energies as:

$$
s=\frac{\beta}{2}\left(U_{\max }-U_{\min }\right)
$$

Equation 24

In the Unilan formalism, $K_{\text {avg }}$ is the Langmuir constant of a hypothetical material with uniform adsorption sites exhibiting the average binding energy, given as:

$$
K_{\text {avg }}=\beta V_{B} e^{-\frac{\beta}{2}\left(U_{\max }+U_{\min }\right)}
$$

Equation 25

Statistical Mechanical Derivation. The statistical mechanical derivation of the Unilan equation is equivalent to that for Case II (MSL) except with a very large number of different single-site partition functions equal to the number of total sites $(\Gamma)$. Each partition function has the same form as Equation 18. The total grand canonical partition function is simply the product:

$$
\Xi(\mu, V, T)=\xi_{1} \xi_{2} \xi_{3} \ldots \xi_{\Gamma}=\prod_{i=1}^{\Gamma} \xi_{i}
$$

Equation 26

The Unilan equation (Equation 23) follows as usual by determining the expected number of adsorbed molecules at constant temperature, volume, and chemical potential. ${ }^{18}$

The generalization of Langmuir's SSL adsorption model to account for different types of adsorption sites (MSL adsorption) permits its application to materials with heterogeneous surfaces. However, the extreme case, a completely amorphous surface where every binding site is different, would require more than $\Gamma$ characteristic parameters to be modelled in this way, an unreasonably large number. The UL equation is an example of a practical solution to this problem, allowing for a wide range of binding energies but enforcing a uniform distribution of them, and thereby minimizing the number of characteristic parameters of the system. The UL model is justified for any system 
exhibiting a distribution of binding sites so long as adsorbate-adsorbate interactions remain insignificant and the bulk gas phase is ideal.

Modern Example: $\mathrm{H}_{2}$ Adsorption on ZTC. A typical characteristic of gas adsorption on amorphous surfaces is a gentle sloping sigmoidal uptake profile when plotted on log-normal axes as a function of pressure. This as demonstrated by $\mathrm{H}_{2}$ adsorption on zeolite-templated carbon (ZTC) 55 at $77 \mathrm{~K}$, as shown in Figure $6 a^{56}$ The experimental data points are each uniquely colored to represent different characteristic binding sites. The amorphous nature of the microporous surface of ZTC leads to a multitude of different binding energies; the average Langmuir constant is $K_{\text {avg }}=2.9 \times 10^{-6} \mathrm{~Pa}^{-1}$. It is clear that no one SSL equation would fit these data over an appreciable range of pressure. The SSL isotherm for a hypothetical material with identical binding sites of the same average binding energy as in the distribution on the surface of ZTC (corresponding to $K_{a v g}$ ) is shown as a purple line and is significantly steeper. A UL model fits the data well (shown as a black line), using only three independent parameters, owing to the fact that $\mathrm{H}_{2}$ has weak intermolecular interactions and that ZTC has no short-range ordered molecular structure. ${ }^{57}$ Owing to its simple functional form yet complex physical representation, the UL model is also successful in engineering applications such as the description of $\mathrm{H}_{2}$ adsorption on MOF-5 across a wide range of temperature and pressure. ${ }^{58}$
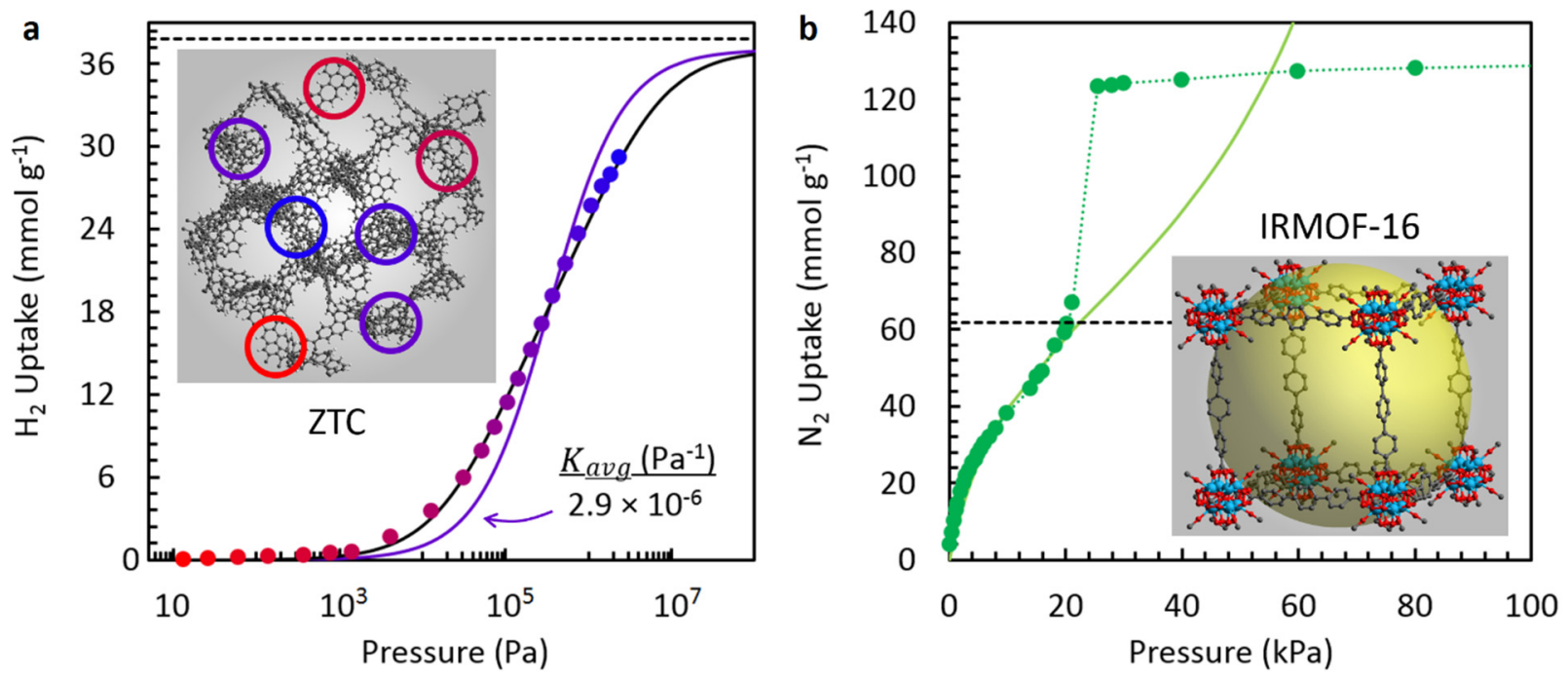

Figure 6. (a) Equilibrium adsorption uptake of $\mathrm{H}_{2}$ on zeolite-templated carbon (ZTC) at $77 \mathrm{~K}, 56$ an example of generalized Langmuir (GL) adsorption. (b) Equilibrium adsorption uptake of $\mathrm{N}_{2}$ simulated on IRMOF-16 at $77 \mathrm{~K}, 59$ an example of multilayer adsorption (MLA) within a porous material.

\section{Case IV: Cooperative Adsorption (CA)}

We return to the case where each binding site on a surface is identical, but now where each site is permitted to host multiple adsorbed molecules. The adsorption of the first molecule on a given site affects the environment (potential energy of adsorption and volume available for translational entropy) of the second adsorbing molecule, and so on as more molecules adsorb on the same site. In other words, adsorption is cooperative since the presence of other adsorbates on the same site affects the energetics of further adsorption. The general case where $n$ neighboring molecules are allowed to interact is complex, and the interested reader is referred elsewhere. ${ }^{53}$ 


\section{Case IV*: Quadratic Adsorption (QA)}

The simplest CA model is that of a surface where each binding site can accommodate up to two adsorbed species, and where an adsorbate-adsorbate intermolecular interaction takes place when both sites are occupied. In this particular case, the quadratic adsorption (QA) isotherm is obtained:

$$
N_{\mathrm{a}}(P)=\Gamma \frac{2 K P+2 K^{2} \phi P^{2}}{1+2 K P+K^{2} \phi P^{2}}
$$

Equation 27

where $K$ is the Langmuir binding constant corresponding to each half-site (identical to Equation 13), $\Gamma$ is the number of adsorption sites (each binding up to two molecules), and $\phi$ is a factor describing the magnitude of adsorbate-adsorbate interactions (when two molecules are bound on a single site). For non-interacting molecules, $\phi=1$ and Equation 27 reduces to the simple Langmuir adsorption isotherm (Equation 7) with a maximum adsorption occupancy of $2 \Gamma$. In the non-trivial case, $\phi>1$ for attractive adsorbate-adsorbate interactions and $\phi<1$ for repulsive adsorbateadsorbate interactions. In the latter case, adsorption of the second molecule is less favored than that of the first, for example due to steric hindrance.

Statistical Mechanical Derivation. The potential energy of each adsorption site depends on its microstate; each site can accommodate up to two molecules and so has three distinct microstates (empty, singly occupied, or doubly occupied). We let $n_{1}, n_{2} \in\{0,1\}$ determine the state of each half of an adsorption site, where $n_{i}=0$ corresponds to the half-site $i$ as empty and $n_{i}=1$ to the half-site as occupied. The potential energy of an individual adsorption site, $U$, is then:

$$
U\left(n_{1}, n_{2}\right)=\left(n_{1}+n_{2}\right) U_{1}+n_{1} n_{2} U_{\mathrm{AA}}
$$

Equation 28

where $U_{1}$ is the spatially uniform potential energy experienced by a single molecule adsorbed on either part of the adsorption site due to its interaction with the surface, and $U_{\mathrm{AA}}$ is the potential energy associated with the interaction between two adsorbed molecules when the site is doubly occupied. The grand canonical partition function for the single adsorption site is then found by summing over all of the microstates (a total of four microstates, with two degenerate singly occupied states):

$$
\begin{aligned}
\xi(\mu, V, T)= & \sum_{n_{1}=0}^{1} \sum_{n_{2}=0}^{1}\left(\Lambda^{-3} V_{B}\right)^{n_{1}+n_{2}} e^{-\beta U\left(n_{1}, n_{2}\right)} e^{\beta \mu\left(n_{1}, n_{2}\right)} \\
& =1+2 \Lambda^{-3} V_{B} e^{-\beta U_{1}} e^{\beta \mu}+\Lambda^{-6} V_{B}^{2} e^{-\beta\left(2 U_{1}+U_{\mathrm{AA}}\right)} e^{2 \beta \mu}
\end{aligned}
$$

The $\Lambda^{-3} V_{B}$ term arises from the configurational part of the partition function where the Boltzmann factor is integrated over all particle coordinates; $V_{B}$ is the free volume offered by each adsorption half-site in the pair. The first, second, and third terms in the sum correspond to the three distinguishable microstates identified above. For $\Gamma$ independent adsorption sites (up to $\Gamma$ pairs of adsorbed molecules), the total grand canonical partition function is factorizable:

$$
\Xi(\mu, V, T)=\xi^{\Gamma}
$$

Equation 30

The expected number of adsorbed molecules is then:

$$
\left\langle N_{\mathrm{a}}\right\rangle=\left(\frac{\partial \log \Xi}{\partial(\beta \mu)}\right)_{\beta, V}=\Gamma \frac{2 \Lambda^{-3} V_{B} e^{-\beta U_{1}} e^{\beta \mu}+2 \Lambda^{-6} V_{B}^{2} e^{-\beta\left(2 U_{1}+U_{\mathrm{AA}}\right)} e^{2 \beta \mu}}{1+2 \Lambda^{-3} V_{B} e^{-\beta U_{1}} e^{\beta \mu}+\Lambda^{-6} V_{B}^{2} e^{-\beta\left(2 U_{1}+U_{\mathrm{AA}}\right)} e^{2 \beta \mu}}
$$


By replacing the chemical potential with the pressure of the ideal gas via Equation 12, the result given above (Equation 27) is achieved with $K=\beta V_{B} e^{-\beta U_{1}}$ as in the SSL model and $\phi=e^{-\beta U_{\mathrm{AA}}}$. Thus if $U_{\mathrm{AA}}<0$ (for attractive interactions), then $\phi>1$, and if $U_{\mathrm{AA}}>0$ (for repulsive interactions), then $\phi<1$, as expected.

\section{Case IV*: Hill Equation}

When each adsorption site is required to be either empty or occupied by the maximum number of occupants, the Hill equation (Equation 6) is obtained. If the maximum number of occupants per site is two, the formal derivation follows that of the more general QA model above, except with only two terms in the single-site partition function:

$$
\xi(\mu, V, T)=1+\Lambda^{-6} V_{B}^{2} e^{-\beta\left(2 U_{1}+U_{\mathrm{AA}}\right)} e^{2 \beta \mu}
$$

Equation 32

The total grand canonical partition function remains factorizable:

$$
\Xi(\mu, V, T)=\xi^{\Gamma}
$$

Equation 33

The Hill equation for $n=2$ follows from the usual derivative:

$$
\left\langle N_{\mathrm{a}}\right\rangle=\left(\frac{\partial \log \Xi}{\partial(\beta \mu)}\right)_{\beta, V}=2 \Gamma \frac{\Lambda^{-6} V_{B}^{2} e^{-\beta\left(2 U_{1}+U_{\mathrm{AA}}\right)} e^{2 \beta \mu}}{1+\Lambda^{-6} V_{B}^{2} e^{-\beta\left(2 U_{1}+U_{\mathrm{AA}}\right)} e^{2 \beta \mu}}
$$

Equation 34

By replacing the chemical potential with the pressure of the ideal gas phase via Equation 12, the following adsorption isotherm (equivalent to Equation 6) is achieved:

$$
\left\langle N_{\mathrm{a}}\right\rangle=2 \Gamma \frac{K^{2} \phi P^{2}}{1+K^{2} \phi P^{2}}
$$

Equation 35

The magnitude of the interaction, $\phi$, is defined as above and the Langmuir constant is defined as in Equation 13. The Hill equation is an over-simplified approximation for most adsorption systems (where any number of molecules between zero and the maximum number of occupants can occupy a given site), but remains a commonly employed model, nevertheless.

Modern Examples. Cooperative adsorption is rare in the modern adsorption literature, and cases of pure QA behavior in a gas-solid system are unknown to the authors. Some representative examples of CA-like behavior are given by $\mathrm{CO}_{2}$ adsorption on the USO-2-M series of MOFs at $298 \mathrm{~K}$, especially in the nickel form $(\mathrm{M}=\mathrm{Ni}) \cdot{ }^{60-61} \mathrm{~A}$ second example is that of ethane on various MOF-74-type materials, especially Mn- and Co-MOF-74, where the maximum occupancy corresponds roughly to two ethane molecules per formula unit.62-63 The adsorption of $\mathrm{CO}_{2}$ on the aluminum form of MIL-91 also exhibits a CA-like inflection. ${ }^{64}$ Lastly, the presence of dynamic moieties within a porous crystal may lead to phenomena resembling CA behavior. ${ }^{65}$

\section{Case V: Dissociative Adsorption (DA)}

In the previous cases, it was assumed that the adsorbate does not undergo any sort of chemical change upon adsorption or desorption. In Case V, Langmuir considered the possibility of adsorption being a two-fold process: residence at the surface adsorption site in combination with molecular dissociation (a result of chemical bonding). This type of behavior commonly occurs at the surface of a catalyst, for example, where a diatomic molecule $\left(\mathrm{A}_{2}\right)$ adsorbs at a binding site and subsequently dissociates into two atoms (A), which are then permitted to diffuse on the surface (freely exploring 
the available adsorption sites as an atomic species). The reverse process requires two neighboring atoms on the surface to reassociate into the diatomic molecule $\left(A+A \rightarrow A_{2}\right)$ and leave the surface (i.e., undergo desorption). In this specific case of homonuclear diatomic/monatomic dissociative adsorption (DA), the adsorption isotherm of the atomic species, $A$, as a function of the pressure of the bulk gaseous molecular species, $\mathrm{A}_{2}$, is:

$$
N_{\mathrm{a}}(P)=\Gamma \frac{K \sqrt{P}}{1+K \sqrt{P}}
$$

Equation 36

Statistical Mechanical Derivation. At thermodynamic equilibrium of the simplest dissociation reaction given above $\left(A_{2} \leftrightarrow A+A\right)$, the chemical potentials must satisfy the following relationship:

$$
2 \mu_{\mathrm{A}}=\mu_{\mathrm{A}_{2}}
$$

Equation 37

That is, the bulk gas phase of the molecular species, $A_{2}$, imposes a chemical potential on the adsorbed atomic species, $A$, at thermodynamic equilibrium. This model does not permit any diatomic $\mathrm{A}_{2}$ to remain adsorbed on the surface; dissociation and adsorption or reassociation and desorption are concomitant in stable equilibrium. Let $U_{1}$ be the spatially homogeneous potential energy experienced by an atomic species $\mathrm{A}$ adsorbed on the surface and $V_{B}$ be the free volume of the binding site (that accommodates a single atom). Then, the single-site grand canonical partition function is:

$$
\xi(\mu, V, T)=1+\Lambda_{\mathrm{A}}^{-3} V_{B} e^{-\beta U_{1}} e^{\beta \mu_{\mathrm{A}}}
$$

Equation 38

The chemical potential and de Broglie wavelength both correspond to the atomic species, A, since it is the relevant species in the adsorbed phase. The total grand canonical partition function for a surface containing $\Gamma$ independent adsorption sites is then:

$$
\Xi(\mu, V, T)=\xi^{\Gamma}
$$

Equation 39

The expected number of monatomic adsorbed species, $A$, is then:

$$
\left\langle N_{\mathrm{a}}\right\rangle=\left(\frac{\partial \log \Xi}{\partial(\beta \mu)}\right)_{\beta, V}=\Gamma \frac{\Lambda_{\mathrm{A}}^{-3} V_{B} e^{-\beta U_{1}} e^{\beta \mu_{\mathrm{A}}}}{1+\Lambda_{\mathrm{A}}^{-3} V_{B} e^{-\beta U_{1}} e^{\beta \mu_{\mathrm{A}}}}
$$

Equation 40

Using Equation 37 and Equation 12 to relate the bulk gas pressure to the chemical potential of the atomic adsorbed species, A, the DA isotherm is obtained (Equation 36). The Langmuir binding constant is explicitly defined as:

$$
K=\Lambda_{\mathrm{A}}^{-3} V_{B} e^{-\beta U_{1}} \sqrt{\Lambda_{\mathrm{A}_{2}}^{3} \beta}
$$

Equation 41

with dimensions of pressure to the power of $1 / 2$, where the pressure refers to the diatomic gas.

Modern Examples. Typical examples of dissociative adsorption (DA) are for simple diatomic molecules like $\mathrm{H}_{2}$ on specific surfaces such as palladium, platinum, tungsten, and silicon (e.g., $\mathrm{H}$ on silicon (111) $7 \times 7$ at $\left.950 \mathrm{~K}^{66}\right)$. A thorough review of hydrogen on silicon is given elsewhere. ${ }^{67}$ The authors are not aware of any strict examples of dissociative adsorption directly on MOF surfaces (without the presence of metal nanoparticles, as in hydrogen spillover68), which has been attributed to the high activation barrier to dissociation. ${ }^{69}$ 


\section{Case VI: Multilayer Adsorption (MLA)}

Lastly, Langmuir addressed the case of multilayer adsorption upon a finite set of underlying adsorption sites on a surface. In the simplest such case, each adsorption site is taken to be identical and independent; after the first molecule is adsorbed on a given site, an additional molecule is permitted to adsorb above it. In this way, despite that the number of adsorption sites is limited, the number of adsorbed molecules is unlimited (assuming a large chemical reservoir is held in contact with the surface at constant chemical potential) and diverges to infinity at the saturation pressure (in the case of subcritical adsorption). Langmuir did not explicitly write a closed-form adsorption isotherm for Case VI, but did remark that such an isotherm would be greatly simplified if the second layer and all above were considered under identical conditions (or even, slightly more complexly, the third layer and all above). ${ }^{3}$

\section{Case VI*: Brunauer-Emmett-Teller (BET) Multilayer Adsorption}

The most well-known multilayer adsorption isotherm is the Brunauer-Emmett-Teller (BET) model; it stipulates that adsorption in the first layer occurs with a constant binding energy and that adsorption in the second, third, and all higher layers occurs with a binding energy equivalent to the energy of condensation (liquefaction) of the bulk gas phase. ${ }^{36}$ Langmuir's more general Case VI predates BET theory by two decades but is in fact inclusive of the BET stipulation. Hence, in this review we highlight the BET equation as a specific example of Case VI since it is the most widely used adsorption isotherm equation, often employed to calculate the specific surface area of porous and nonporous materials. In the BET model, each binding site can accommodate an "adsorption column" consisting of a single adsorbed molecule at the base and up to an infinite number of adsorbed molecules above it. Adsorbate-adsorbate interactions within each column are ignored, as well as interactions between adsorption sites. For a surface containing $\Gamma$ adsorption sites, the BET multilayer adsorption isotherm is:

$$
N_{\mathrm{a}}(P)=\Gamma \frac{K P}{\left(1-K_{l} P\right)\left(1+\left(K-K_{l}\right) P\right)} \quad \text { Equation } 42
$$

As usual, $K$ is the Langmuir binding constant describing the interaction between the adsorbate in the first monolayer and the solid surface; $K_{l}$ is an analogous binding constant describing the affinity of an adsorbate in one of the higher layers toward the adsorbate molecule below it (taken to be equivalent to that of bulk liquefaction).

Statistical Mechanical Derivation. As for the QA model, the potential energy of adsorption must first be expressed as a function of each microstate of a given adsorption site. As before, let $U_{1}$ be the potential energy of a molecule adsorbed in the first layer, directly interacting with the adsorbent surface. In all subsequent layers, the potential energy associated with adsorption is $U_{l}$, a consequence of interacting with the gas molecule in the layer below. There is an infinite set of microstates for each adsorption site: empty, singly occupied, doubly occupied, and so on, up to infinitely occupied. With $n$ as the number of molecules adsorbed on an adsorption site, the potential energy of a single column of molecules as a function of its microstate is:

$$
U(n)= \begin{cases}0 & \text { if } n=0 \\ U_{1}+(n-1) U_{l} & \text { if } n \in\{1,2, \ldots\}\end{cases}
$$

Equation 43 
The grand canonical partition function of a single adsorption site (column) is then a sum over all possible microstates including the empty state $(n \in\{0,1,2, \ldots\})$ :

$$
\xi(\mu, V, T)=1+q_{1} e^{\beta \mu}+q_{1} q_{l} e^{2 \beta \mu}+q_{1} q_{l}^{2} e^{3 \beta \mu}+\cdots
$$

Equation 44

The canonical partition functions of a single molecule adsorbed on the first layer and in subsequent layers are, respectively:

$$
\begin{aligned}
& q_{1}=\Lambda^{-3} V_{B} e^{-\beta U_{1}} \\
& q_{l}=\Lambda^{-3} V_{B, l} e^{-\beta U_{l}}
\end{aligned}
$$

Equation 45

Equation 46

By factoring out the contribution in each term in Equation 43 from the molecule in the first layer, we recognize the geometric series:

$$
\xi(\mu, V, T)=1+q_{1} e^{\beta \mu}\left(1+q_{l} e^{\beta \mu}+\left(q_{l} e^{\beta \mu}\right)^{2}+\cdots\right)=1+\frac{q_{1} e^{\beta \mu}}{1-q_{l} e^{\beta \mu}} \quad \text { Equation } 47
$$

The total grand canonical partition function for a surface containing $\Gamma$ independent adsorption sites is factorizable:

$$
\Xi(\mu, V, T)=\xi^{\Gamma}
$$

Equation 48

The total expected number of adsorbed molecules is then:

$$
\left\langle N_{\mathrm{a}}\right\rangle=\left(\frac{\partial \log \Xi}{\partial(\beta \mu)}\right)_{\beta, V}=\Gamma \frac{q_{1} e^{\beta \mu}}{\left(1-q_{l} e^{\beta \mu}\right)\left(1+\left(q_{1}-q_{l}\right) e^{\beta \mu}\right)} \quad \text { Equation } 49
$$

Replacing chemical potential in Equation 49 with the pressure of the bulk gas phase via Equation 12, we arrive at the BET adsorption isotherm (Equation 42) with $K$ defined as in Equation 13 and:

$$
K_{l}=\beta V_{B, l} e^{-\beta U_{l}}
$$

Equation 50

Modern Example: $\mathrm{N}_{2}$ Adsorption on IRMOF-16. A representative example of BET adsorption within a porous material is $\mathrm{N}_{2}$ adsorption on the surface of large-pore MOFs such as IRMOF-12, -14, and -16, where the latter is shown in Figure 6 b. ${ }^{59}, 70$ The $\sim 2 \mathrm{~nm}$ wide pores of IRMOF-16 can accommodate multilayer adsorption over a limited pressure range up to $\sim 20 \mathrm{kPa}$ when cross-pore interactions become significant. This effect is obvious at higher pressures where the measured adsorption uptake departs dramatically from the expected uptake, first toward higher uptake as pore filling completes (exhibiting strong intermolecular interactions between multilayers on both sides of the pore) and then showing abrupt filling and no further adsorption. Similar MLA isotherms have been measured for $\mathrm{N}_{2}$ adsorption on other large-pore MOFs such as NU-100,71 NU-1300,72 and NU-109 and NU-11073.

Below the "roughening temperature," layer-by-layer adsorption on crystalline surfaces is distinctly observed to occur, resulting from significantly different binding interactions characteristic of each layer (e.g., Xe adsorption on palladium (100) between 60-70 $\mathrm{K}^{74}$ ). Such an adsorption system is accurately described by a more complex MLA equation than that of the BET model, with individual Langmuir constants assigned to each layer. 


\section{Applications and Extensions of Langmuir's Theory}

The Langmuir theory of adsorption is useful for modeling and designing engineering processes, both qualitatively and quantitatively. Since each Langmuir model is founded on reasonable physical assumptions, thermodynamic consistency is also inherent to each adsorption isotherm, and the calculation of thermodynamic quantities such as the enthalpy of adsorption is direct and analytical. The most common material properties calculated via Langmuir's theory are the specific surface area and the isosteric enthalpy of adsorption. Two common engineering processes of interest to gas-solid adsorption systems are storage and separation.

Surface Area Measurements. Specific surface area is an important characteristic of porous materials, especially for determining the applicability of a material for engineering applications such as storage or separation. The two most widely used equations are the BET and SSL models, the latter of which was first used by Langmuir himself in $1918^{3}$. While neither model is strictly appropriate for the description of gas adsorption within narrow micropores, it has been shown that the BET model is often suitable for the estimation of the true surface area of microporous and mesoporous materials including MOFs and zeolites (despite that the number of multilayers is constrained) when proper consistency criteria ${ }^{75}$ are employed in determining the range of partial pressure over which to fit the data. ${ }^{59,76-77}$

The BET formalism prevalent in the literature is written as follows:

$$
N_{\mathrm{a}}(P)=\Gamma \frac{c\left(P / P_{0}\right)}{\left(1-P / P_{0}\right)\left(1+(c-1) P / P_{0}\right)}
$$

Equation 51

where $P_{0}$ is the saturation pressure of the adsorbate, defined as:

$$
P_{0}=\frac{P}{q_{l} e^{\beta \mu}}=K_{l}^{-1}
$$

Equation 52

and $c$ is the BET constant, defined as:

$$
c=\frac{q_{1}}{q_{l}}
$$

Equation 53

A common linearized rearrangement, where B is simply the "BET variable", is:

$$
B=\frac{P / P_{0}}{N_{\mathrm{a}}\left(1-P / P_{0}\right)}=\frac{1}{\Gamma c}+\frac{c-1}{\Gamma c}\left(P / P_{0}\right)
$$

Equation 54

Brunauer, Emmett, and Teller described three regions along the BET isotherm in Equation 54: a concave region at low pressure, a convex region at high pressure, and a linear region at intermediate pressure. They originally specified the relative pressure $\left(P / P_{0}\right)$ range of $0.05-0.3$ as the linear region from which the number of surface sites, $\Gamma$, can be dependably extracted. In the case of high surface area, microporous materials such as zeolites and MOFs, however, this range has proven to be inadequate as a universal standard and a now widely accepted set of self-consistency criteria have been proposed. ${ }^{75}$ Once the linear range is determined and $\Gamma$ is extracted, the BET surface area, $S A_{B E T}$, is calculated as:

$$
S A_{B E T}=\Gamma \cdot S A_{B}
$$

Equation 55 
where $S A_{B}$ is the surface area of a single binding site. The conventional cross-sectional area of a $\mathrm{N}_{2}$ molecule for BET surface area calculation is $0.162 \mathrm{~nm}^{2}$, which allows researchers across different laboratories to have a standard for materials comparison. For example, in the case of IRMOF-16, the BET surface area (monolayer capacity, shown as a dashed black line in Figure 6b) determined herein corresponds to $61.8 \mathrm{mmol} \mathrm{g}^{-1}$ or $6030 \mathrm{~m}^{2} \mathrm{~g}^{-1}$. This is consistent with the geometrical surface area of the crystal ( $\sim 600 \mathrm{~m}^{2} \mathrm{~g}^{-1}$ as determined by a Monte Carlo integration technique $\left.{ }^{59}\right)$. It should be noted that challenges still persist in using the BET model to accurately estimate the monolayer capacity of porous materials, especially those with a diversity of pore sizes. ${ }^{77}$

Enthalpy of Adsorption. Another important characteristic for determining the applicability of a porous material to storage and separation processes is the adsorption binding strength. While the binding energy (or energies, in the case of a multi-site adsorption model) could in principle be directly extracted from any of Langmuir's simple models via the relationship in Equation 13, a more general (less model-dependent) approach is to calculate the difference in enthalpy between the adsorbed phase and the bulk gas phase, $\Delta H_{a d s}$, via the Clapeyron equation or a variant thereof.78-79 When the bulk gas phase is ideal, the rearranged Clausius-Clapeyron equation is employed:

$$
\Delta H_{a d s}=H_{\mathrm{a}}-H_{\mathrm{g}}=T \Delta S_{a d s}=-\frac{R T^{2}}{P}\left(\frac{d P}{d T}\right)_{N_{\mathrm{a}}}
$$

Equation 56

This requires the measurement of multiple isotherms at different temperatures, and investigation of the temperature-pressure relationship along a line of constant adsorbed amount (an isostere). The resulting thermodynamic quantity is referred to as the isosteric enthalpy (or often "isosteric heat") of adsorption; for SSL adsorption under a bulk gas phase that is ideal, it is equal to the negative of the binding energy (i.e., $\Delta H_{a d s}=-U_{1}$ ). The SSL, DSL, and UL models are routinely employed to interpolate measured data, an important step in the calculation of the isosteric enthalpy of adsorption. 58,80 When a sufficient quantity of data are measured, the enthalpy of adsorption measured by the sorption isosteric method compares reasonably accurately to calorimetric measurements. ${ }^{81}$ It should be noted that there remains debate as to the validity of the isosteric method in the high pressure (non-ideal gas) regime where the measured quantity of adsorption (the "Gibbs surface excess") departs from the actual quantity; under such conditions, a so-called "isoexcess" method has been proposed which does not rely on the use of any adsorption model. $82-83$

Deliverable Gas Storage. The key material design metric for adsorptive storage is the deliverable capacity: the amount adsorbed at the storage pressure, $P_{H}$, minus that remaining in the adsorbed phase when the pressure is below the useful threshold, $P_{L}$ (e.g., when the storage vessel can no longer supply a fuel cell or combustion engine). For example, the US Department of Energy (DOE) target for natural gas storage on-board mobile vehicles sets $P_{H}=6.5 \mathrm{MPa}$ and $P_{L}=0.5 \mathrm{MPa}$ for delivery at room temperature. In an adsorption system characterized by SSL theory (Equation 7), the deliverable capacity, $N_{\text {del }}$, for an isothermal pressure swing between $P_{H}$ and $P_{L}$ is:

$$
N_{\text {del }}=\Gamma\left(\frac{K P_{H}}{1+K P_{H}}-\frac{K P_{L}}{1+K P_{L}}\right)
$$

Equation 57

For a given storage and delivery pressure, the deliverable capacity depends importantly on the material properties, $K$ and $\Gamma$. When the number of adsorption sites, $\Gamma$, is fixed (i.e., maximized), the optimal Langmuir constant to maximize $N_{\text {del }}$ is: 


$$
K_{o p t}=\frac{1}{\sqrt{P_{H} P_{L}}}
$$

Equation 58

If the binding energy is too weak, corresponding to $K<K_{o p t}$, the low storage capacity at the storage pressure, $P_{H}$, limits the deliverable amount of adsorbed gas. On the other hand, if the binding energy is too strong, corresponding to $K>K_{\text {opt }}$, a significant quantity of adsorbate remains bound at the minimum discharge pressure, $P_{L}$, limiting the deliverable capacity as well. These effects are optimally balanced for a material that exhibits a Langmuir constant given by $K=K_{\text {opt }}$. Then, by assuming a typical entropy of adsorption based on the difference in entropy between the bulk gas and the adsorbed phases, this analysis can be used to estimate the optimal enthalpy of adsorption to maximize the deliverable storage capacity. ${ }^{84}$ Several MOFs have been identified by a similar method as record-holding materials for both methane ${ }^{85}$ and hydrogen ${ }^{86}$ storage at ambient conditions, and the search continues for higher capacity materials using a design-driven approach.

Mixed (Competitive) Adsorption. Another chief application of porous materials is to separate gaseous mixtures through selective adsorption. Hence, it is useful to consider the case of mixed gas adsorption where two different adsorbate species compete for the same set of adsorption sites. Consider a surface comprising $\Gamma$ adsorption sites exposed to an ideal gas mixture $(A+B)$. If the $A$ and $B$ molecules are comparable in size so that each adsorption site accommodates only a single $A$ or B molecule, the adsorption isotherm (where $N_{\mathrm{a}}$ is the total number of A and B molecules) is:

$$
N_{\mathrm{a}}(P)=\Gamma \frac{K_{\mathrm{A}} P_{\mathrm{A}}+K_{\mathrm{B}} P_{\mathrm{B}}}{1+K_{\mathrm{A}} P_{\mathrm{A}}+K_{\mathrm{B}} P_{\mathrm{B}}}
$$

Equation 59

where $K_{\mathrm{A}}$ and $K_{\mathrm{B}}$ are the Langmuir constants corresponding to the binding of species $\mathrm{A}$ and $\mathrm{B}$, and $P_{\mathrm{A}}$ and $P_{\mathrm{B}}$ are the partial pressures of species in the mixed gas phase (of total pressure $P$ ). ${ }^{87}$ The statistical mechanical derivation uncovers the dependence of the Langmuir constant on the volume of the binding site, $V_{B, k}$, and binding energy, $U_{k}$, where both characteristics vary depending on the species $(k)$ occupying the adsorption site, as well as the temperature. Thereby, the ratio of the number of adsorption sites occupied by species A to species $B$ at dilute conditions is:

$$
\frac{N_{\mathrm{a}, \mathrm{A}}}{N_{\mathrm{a}, \mathrm{B}}}=\frac{K_{\mathrm{A}} P_{\mathrm{A}}}{K_{\mathrm{B}} P_{\mathrm{B}}}=\frac{P_{\mathrm{A}}}{P_{\mathrm{B}}} \frac{V_{B, \mathrm{~A}}}{V_{B, \mathrm{~B}}} e^{-\beta\left(U_{\mathrm{A}}-U_{\mathrm{B}}\right)}
$$

Equation 60

which depends on the ratio of partial pressures, on the ratio of free volume available to each adsorbate (an entropic effect), and exponentially on the difference in binding energies. As in the DSL model, lower temperature serves to amplify the occupancy ratio dependence on the difference in potential energies of adsorption of the two species. A representative example of the direct application of Equation 59 to measurements of mixed-adsorption equilibria is found in the adsorption of binary mixtures of $\mathrm{CO}_{2}, \mathrm{H}_{2}$, and $\mathrm{N}_{2}$ on activated carbon. 88

The measurement of mixed-adsorption equilibria requires an additional step over pure adsorption measurements: varying the composition of the bulk (gaseous or supercritical) fluid and then obtaining the corresponding composition of the adsorbed phase, which is time-intensive and technically complicated. ${ }^{99}$ To circumvent these limitations, adsorption selectivity is very often estimated by comparing the pure gas adsorption equilibria of each of the components of the desired mixture and applying a simple model. Ideal adsorbed solution theory (IAST) ${ }^{90}$ is a simple model for describing the mixed adsorption state based on the pure component adsorption equilibria, analogous to Raoult's law for ideal vapor/liquid solutions. The main principle lies in the concept of 
a constant spreading pressure of the adsorbed phase at equilibrium, wherein all adsorbate species are assumed to be non-interacting. From the pure-state adsorption isotherms, $n_{k}^{\circ}\left(P_{k}^{\circ}, T\right)$, the spreading pressure, $\pi_{k}$, is estimated for each component as a function of the pressure of the pure bulk phase above it, $P_{k}^{\circ}$; in turn, this relationship is extended to the co-adsorption state by assuming that the (individual) adsorbed phase of each component will behave the same as a function of the partial pressure of that component in the mixed state. The binary mixed-state total adsorption isotherm, $n_{\mathrm{a}}\left(\left\{P_{\mathrm{A}}, P_{\mathrm{B}}\right\}, T\right)$, for a given gas phase composition defined by $\left\{P_{\mathrm{A}}, P_{\mathrm{B}}\right\}$ can therefore be estimated in addition to the individual (mixed-state) adsorption isotherms of each component, $n_{k}\left(\left\{P_{\mathrm{A}}, P_{\mathrm{B}}\right\}, T\right)$.

The results of IAST analysis are remarkably accurate when compared to experimental mixedadsorption equilibria ${ }^{91-92}$ and also when IAST is applied to Grand Canonical Monte Carlo (GCMC) pure-adsorption simulations and compared to mixed-adsorption GCMC simulations ${ }^{93-94}$. However, the overall accuracy of the method is dependent on the similarity of the components of the adsorbed phase as well as the binding strength toward and homogeneity of the adsorbent surface; corrections can be applied for systems where the co-adsorbed phase is significantly non-ideal, referred to as RAST.95-96 The analysis of numerous adsorption pairs and mixtures have been carried out using IAST, demonstrating extraordinary prospects for using MOFs, ${ }^{97}$ zeolites, and other designable porous materials as solid-phase separation media: e.g., as in carbon capture,98-99 hydrogen recycling, ${ }^{100}$ hydrocarbon separations, ${ }^{62,101}$ and harmful gas separations from air, 102 among many other applications.

Flexible, Gas-Responsive Materials. It is usually assumed that the adsorption of gas molecules does not change the thermodynamic properties or structure of the adsorbent itself. Such a guest-host perspective is a simplification that is not applicable in porous materials that undergo structural changes induced by the adsorption of gas molecules: so-called "flexible," "soft," or "breathing" materials. ${ }^{103-105}$ The structural changes in flexible MOFs often have a profound influence on adsorption and can lead to counterintuitive properties such as negative gas adsorption steps. ${ }^{106-107}$ The Langmuir model serves as a platform onto which further complexity, such as flexibility, can be integrated.

Gate-opening materials exist in a collapsed, nonporous state in vacuum that persists even when they are exposed to low pressures (low chemical potential) of the gaseous adsorbate. ${ }^{108}$ As the chemical potential of the gaseous reservoir is increased, at some condition the material abruptly expands to a porous state and an adsorption step occurs. A flagship example of gate-opening behavior is in the adsorption of $\mathrm{CH}_{4}$ on $\mathrm{Co}(\mathrm{bdp})$ (where bdp ${ }^{2-}=1,4$-benzenedipyrazolate). ${ }^{109} \mathrm{At}$ room temperature, gate opening occurs concomitant with an abrupt step at 1.6 MPa on the adsorption branch of the $\mathrm{CH}_{4}$ uptake isotherm; the reverse transition is detected at $0.7 \mathrm{MPa}$ on the desorption branch. Importantly, this step occurs between the desired pressure swing range involved in vehicular adsorbed natural gas storage, thereby imparting Co(bdp) with an extremely high deliverable capacity of $\mathrm{CH}_{4}: N_{\text {del }}=197 \mathrm{~L}_{\text {SтP L}} \mathrm{L}^{-1}\left(\sim 10 \mathrm{mmol} \mathrm{g}^{-1}\right)$. Moreover, the structural transition in $\mathrm{Co}(\mathrm{bdp})$ is endothermic, offsetting a portion of the heat of adsorption and mitigating storage inefficiencies upon refilling.

Breathing materials, like gate-opening materials, exhibit two structural forms that are stable under different conditions: a narrow-pore and large-pore state. The large-pore state of a breathing material is stable in vacuum and up to low pressures (low chemical potential) of the gaseous reservoir). At an intermediate transition pressure, the large-pore state collapses to the narrow- 
pore state, affording a stronger adsorption interaction. At a higher transition pressure, the framework expands again to the large-pore state, accommodating more adsorbed molecules with a weaker characteristic binding energy. A flagship example of breathing behavior is in $\mathrm{CO}_{2}$ adsorption on M-MIL-53 ( $\mathrm{M}=\mathrm{Cr}, \mathrm{Al}) .{ }^{110-111}$ At room temperature on Al-MIL-53, the first abrupt step is centered at $24 \pm 15 \mathrm{kPa}$ and the second at $425 \pm 75 \mathrm{kPa}$ where the pressure range corresponds to the width of the hysteresis loop. ${ }^{111}$

A general statistical mechanical treatment of gate-opening and breathing in gas-solid adsorption systems has been developed.112 Instead of employing the grand canonical ensemble as in the above six derivations (where the adsorbed phase is held at constant $\mu, V$, and $T$ ), the osmotic ensemble is preferred (at constant $\mu, P$, and $T$ ). In this treatment, the chemical potential imposed by the bulk gas phase can be decoupled from the mechanical pressure so that the effects of applying mechanical force on the solid adsorbent can be studied. Such experiments have been performed with Co(bdp), illustrating that the application of mechanical force can serve to collapse the porous state of a gateopening material and expel the adsorbed gas. The two "rigid host" adsorption systems for the two distinct states of the gate-opening adsorbent could be accurately modeled by SSL equations. ${ }^{109}$

Structural transitions of adsorbent materials in the presence of gaseous adsorbate may also occur without any expansion or contraction of the unit cell. A representative example of such behavior is that of Xe adsorption on $\mathrm{Ni}\left(\mathrm{SiF}_{6}\right)(\mathrm{pyz})_{2}(\mathrm{pyz}=$ pyrazine), a pillared square grid MOF with rotatable pyrazine struts. ${ }^{113}$ The Xe adsorption isotherm at room temperature exhibits a pronounced inflection at $\sim 50 \mathrm{kPa}$; in situ X-ray diffraction measurements, density functional theory calculations, and classical Monte Carlo molecular simulations indicate that the inflection is a result of the rotational states of the pyrazine ligands organizing to achieve a more favorable gas-host interaction. A simple statistical mechanical model of porous MOFs with rotating ligands was subsequently developed which could accurately reproduce the adsorption inflection observed in experiment. 65 The model is an extension of the SSL model with additional complexity to account for the two states of the rotating ligands, which modifies the adsorption binding interaction. Studies of gate-opening, breathing, and rotating ligand behavior are leading to important adsorbent material design principles aimed at exploiting the sigmoidal (S-shaped) isotherm to maximize deliverable adsorption capacity and separation selectivity.

\section{Conclusions}

The legacy of Irving Langmuir is widespread, from biology to atmospheric science, but no field is more closely associated with his name than adsorption: the science of interfaces. In the century since the publication of his simple theory of gas-solid adsorption, there has never been a time when his work was more hungrily consumed (i.e., referenced in the scientific literature) than it is now. Despite that Langmuir's six adsorption classifications were initially developed to treat gas adsorption on planar, external surfaces (with the exception of Case III where Langmuir remarks about applicability to porous materials), recently discovered classes of nanostructured and porous materials now dominate the landscape of gas-solid adsorption studies. Most notably, metal-organic frameworks (MOFs) exhibit widely tunable crystalline environments for binding adsorbates (e.g., strongly binding open-metal sites and weakly binding organic linker and pocket sites), in addition to internal porosities with unprecedented surface areas for adsorption (e.g., $>6000 \mathrm{~m}^{2} \mathrm{~g}^{-1}$ ). Porous framework carbon-based materials such as zeolite-templated carbon (ZTC) ${ }^{55}$ and microporous silicates (zeolites) ${ }^{114}$ also present unusual and highly interesting environments for gas, liquid, and ion adsorption. This type of adsorption in confined "nanospaces", where a potential energy field for 
adsorption is created in a three-dimensional space, brings a new level of complexity and richness to studies of adsorption. The limitations of Langmuir's theory in treating complex adsorbents have, of course, long been noted. ${ }^{115}$ It is often appropriate to employ a potential theory of adsorption in such systems, ${ }^{116-118}$ or a more complex model such as one based on density functional theory ${ }^{119-120}$ or molecular simulations ${ }^{121}$; nevertheless, the Langmuir model still serves as a robust, simple platform onto which further complexity can easily be integrated to meet the needs of both routine and unusual problems in adsorption science. The fundamental theories of monolayer and multilayer adsorption, both attributed to Langmuir's pivotal 1916-1918 works, remain widely used today. 


\section{Associated Content}

\section{Nomenclature}

The nomenclature used in Langmuir 1918 has not been adopted in this review in favor of more modern nomenclature and statistical mechanical conventions, as shown below. The phase of the adsorptive molecule " $A$ " is indicated by either " $g$ " for gas or " $a$ " for adsorbed. The use of the term "gas" or phase " $g$ " is inclusive of supercritical fluids.

\begin{tabular}{|c|c|c|c|}
\hline Symbol & Quantity & Typical Units & Langmuir's Symbol ${ }^{3}$ \\
\hline$r$ & surface rate & $\mathrm{m}^{-2} \mathrm{~s}^{-1}$ & $\nu, \mu$ \\
\hline$p$ & probability & (dimensionless) & $\alpha$ \\
\hline$\theta$ & fractional site occupancy & (dimensionless) & $\theta_{i}$ \\
\hline-- & relative adsorption lifetime & $\mathrm{m}^{2} \mathrm{~s}$ & $\sigma$ \\
\hline$N$ & number & (dimensionless) & $N$ \\
\hline$n$ & mole number & mol & $\eta$ \\
\hline$T$ & temperature & $\mathrm{K}$ & $T$ \\
\hline$P$ & pressure & $\mathrm{Pa}$ & $p$ \\
\hline$V$ & volume & $\mathrm{m}^{3}$ & $V$ \\
\hline$m$ & molecular mass & $\mathrm{kg}$ & $M$ \\
\hline$R$ & gas constant & $\mathrm{J} \mathrm{K}^{-1} \mathrm{~mol}^{-1}$ & $R$ \\
\hline$K$ & Langmuir binding constant & $\mathrm{Pa}^{-1}$ & $a_{i}$ \\
\hline$\Gamma$ & Langmuir scaling constant & $\mathrm{mmol} \mathrm{g}^{-1}$ & $b_{i}$ \\
\hline$A$ & exponential prefactor & varies & -- \\
\hline$\alpha$ & weighting constant & (dimensionless) & $\beta_{i}$ \\
\hline$n$ & Hill interaction constant & (dimensionless) & -- \\
\hline$B$ & BET variable & $\mathrm{g} \mathrm{mmol}^{-1}$ & -- \\
\hline$c$ & BET constant & (dimensionless) & -- \\
\hline$N_{\mathrm{a}}$ & number adsorbed & (dimensionless) & \\
\hline$\Gamma$ & Langmuir scaling constant & $g^{-1}$ & \\
\hline$U$ & adsorption site binding energy & $\mathrm{J}$ & \\
\hline$\mu$ & chemical potential & $\mathrm{J}$ & \\
\hline$q, \xi$ & single-site partition function & (dimensionless) & \\
\hline$Q, \Xi$ & total partition function & (dimensionless) & \\
\hline$\Lambda$ & thermal de Broglie wavelength & $\mathrm{m}$ & \\
\hline$\pi$ & spreading pressure & $\mathrm{N} \mathrm{m}^{-1}$ & \\
\hline
\end{tabular}




\section{Supporting Information}

The Supporting Information is available free of charge via the Internet at http://pubs.acs.org. Experimental Notes and Kinetic and Statistical Mechanical Derivations of Langmuir's Theory.

\section{Author Information}

\section{Corresponding Authors}

*Email: nicholas.stadie@montana.edu

ORCID

Nicholas P. Stadie: 0000-0002-1139-7846

\section{Notes}

The authors declare no competing financial interest.

\section{Acknowledgements}

We thank Cory Simon and Arni Sturluson for invaluable contributions to the statistical mechanical derivations and acknowledge several important historical ${ }^{6-8}$ and biographical ${ }^{24,122-125}$ references used extensively in the preparation of this manuscript. 


\section{References}

1. Langmuir, I., The Constitution and Fundamental Properties of Solids and Liquids. Part I. Solids. J. Am. Chem. Soc. 1916, 38 (11), 2221-2295.

2. Langmuir, I., The Constitution and Fundamental Properties of Solids and Liquids. II. Liquids. J. Am. Chem. Soc. 1917, 39(9), 1848-1906.

3. Langmuir, I., The Adsorption of Gases on Plane Surfaces of Glass, Mica and Platinum. J. Am. Chem. Soc. 1918, 40 (9), 1361-1403.

4. $\quad$ Nobel Lectures: Chemistry 1922-1941. Elsevier Publishing Company: Amsterdam, 1966. 5. Furukawa, H.; Cordova, K. E.; O’Keeffe, M.; Yaghi, O. M., The Chemistry and Applications of Metal-Organic Frameworks. Science 2013, 341 (6149), 1230444.

6. $\quad$ Rouquerol, F.; Rouquerol, J.; Sing, K. S. W., Adsorption by Powders and Porous Solids: Principles, Methodology, and Applications. Academic Press: San Diego, 1999; p xvi, 467 p.

7. Dąbrowski, A., Adsorption-from Theory to Practice. Adv. Colloid Interface Sci. 2001, 93 (13), 135-224.

8. Kiefer, S.; Robens, E., Some Intriguing Items in the History of Volumetric and Gravimetric Adsorption Measurements. J. Therm. Anal. Calorim. 2008, 94 (3), 613-618.

9. Kayser, H., Ueber Die Verdichtung Von Gasen an Oberflächen in Ihrer Abhängigkeit von Druck und Temperatur. Annalen der Physik 1881, 250 (11), 450-468.

10. McBain, J. W., XCIX. The Mechanism of the Adsorption ("Sorption") of Hydrogen by Carbon. The London, Edinburgh, and Dublin Philosophical Magazine and Journal of Science 1909, 18(108), 916-935.

11. Gibbs, J. W., On the Equilibrium of Heterogeneous Substances (Part II). Trans. Conn. Acad. 1877, III, 343-524.

12. Henneberg, W.; Stohmann, F., Ueber das Verhalten der Ackerkrume Gegen Ammoniak und Ammoniaksalze. Justus Liebigs Annalen der Chemie 1858, 107 (2), 152-174.

13. Chappuis, P., Ueber die Verdichtung der Gase auf Glasoberflächen. Annalen der Physik1879, 244 (9), 1-29.

14. Joulin, M. L., Recherches Expérimentales Sur La Diffusion. Annales de Chimie et de Physique 1881, 5 (23), 398-421.

15. Favre, P. A., Sur la Condensation des Gaz par les Corps Solides et sur la Chaleur Dégagée dans l'Acte de cette Absorption - sur les Relations de ces Effets avec les Chaleurs de Liquéfaction ou de Solidification des Gaz. C. R. Acad. Sci.1854, 39(16), 729-733.

16. Favre, P. A., Recherches Thermiques sur la Condensation des Gaz par les Corps Solides et la Chaleur Dégagée dans l'Acte de cette Absorption - Relations de ces Effets avec les Chaleurs de Liquéfaction et de Solidification des Gaz. Ann. Chim. Phys. 1874, 1 (5), 209-261.

17. Freundlich, H., Über die Adsorption in Lösungen. Z. Phys. Chem. 1907, 57(1), 385-470.

18. Sips, R., On the Structure of a Catalyst Surface. J. Chem. Phys. 1948, 16 (5), 490-495.

19. Sips, R., On the Structure of a Catalyst Surface. II. J. Chem. Phys. 1950, 18 (8), 1024-1026.

20. Polanyi, M., Über die Adsorption vom Standpunkt des Dritten Wärmesatzes. Verh. Dtsch.

Phys. Ges. 1914, 16, 1012-1016.

21. Polanyi, M., Adsorption von Gasen (Dämpfen) Durch ein Festes Nichtflüchtiges Adsorbens. Verh. Dtsch. Phys. Ges. 1916, 18, 55-80.

22. Polanyi, M.; London, F., Über die Atomtheoretische Deutung der Adsorptionskräfte. Naturwissenschaften 1930, 18 (50), 1099-1100.

23. Polanyi, M., The Potential Theory of Adsorption. Science 1963, 141 (3585), 1010-1013.

24. $\quad$ Rosenfeld, A., Men of Physics: Irving Langmuir. Pergamon Press Ltd.: 1966.

25. Langmuir, I. Ueber Partielle Wiedervereinigung Dissociierter Gase im Verlauf einer Abkühlung. Göttingen, 1906. 
26. Langmuir, I., The Dissociation of Water Vapor and Carbon Dioxide at High Temperatures. J. Am. Chem. Soc. 1906, 28 (10), 1357-1379.

27. Langmuir, I., Chemical Reactions at Low Pressures. J. Am. Chem. Soc. 1915, 37(5), 1139-

1167.

28. Langmuir, I., The Evaporation, Condensation and Reflection of Molecules and the Mechanism of Adsorption. Phys. Rev. 1916, 8(2), 149.

29. Langmuir, I., The Vapor Pressure of Metallic Tungsten. Phys. Rev. 1913, 2 (5), 329.

30. Langmuir, I., A Chemically Active Modification of Hydrogen. J. Am. Chem. Soc. 1912, 34 (10), 1310-1325.

31. Roberts, G., Langmuir-Blodgett Films. Plenum Press: New York, NY, 1990.

32. Ulman, A., An Introduction to Ultrathin Organic Films: From Langmuir-Blodgett to SelfAssembly. Academic Press Inc.: San Diego, CA, 1991.

33. Langmuir, I., The Condensation Pump: An Improved Form of High Vacuum Pump. Journal of the Franklin Institute 1916, 182 (6), 719-743.

34. Roth, A., Vacuum Technology. Elsevier: New York, 2012.

35. Gaines, G. L.; Wise, G., Insiders, Outsiders, and Surfaces: Irving Langmuir's Contribution to Catalysis. Heterogeneous Catalysis, Davis, B. H.; Hettinger, W. P., Eds. American Chemical Society, 1983, 222, 13-22.

36. Brunauer, S.; Emmett, P.; Teller, E., Adsorption of Gases in Multimolecular Layers. J. Am. Chem. Soc. 1938, 60, 309-319.

37. Brunauer, S., About Some Critics of the BET Theory. Langmuir 1987, 3, 3-4.

38. Adamson, A. W.; Gast, A. P., Physical Chemistry of Surfaces. John Wiley \& Sons, Inc.: New York, 1967.

39. Barcroft, J.; Camis, M., The Dissociation Curve of Blood. J. Physiol. 1909, 39(2), 118-142.

40. Bohr, C.; Hasselbalch, K.; Krogh, A., Ueber einen in Biologischer Beziehung Wichtigen

Einfluss, den die Kohlensäurespannung des Blutes uuf dessen Sauerstoffbindung übt.

Skandinavisches Archiv für Physiologie 1904, 16 (2), 402-412.

41. Hill, A. V., The Mode of Action of Nicotine and Curari, Determined by the Form of the Contraction Curve and the Method of Temperature Coefficients. J. Physiol. 1909, 39(5), 361-373.

42. Hill, A. V., The Possible Effects of the Aggregation of the Molecules of Haemoglobin on Its Dissociation Curves. J. Physiol. 1910, 40, 4-7.

43. Edsall, J. T. In Hemoglobin and the Origins of the Concept of Allosterism, Federation proceedings, 1980; pp 226-235.

44. Garrett, R. H.; Grisham, C. M., Biochemistry. 5th ed.; Brooks/Cole, Cengage Learning: Boston, 2010.

45. Rossi-Fanelli, A.; Antonini, E., Studies on the Oxygen and Carbon Monoxide Equilibria of Human Myoglobin. Arch. Biochem. Biophys. 1958, 77 (2), 478-492.

46. Ferry, R. M.; Green, A. A., Studies in the Chemistry of Hemoglobin. III. The Equilibrium between Oxygen and Hemoglobin and Its Relation to Changing Hydrogen Ion Activity. J. Biol. Chem. 1929, $81(1), 175-203$.

47. Pauling, L., The Oxygen Equilibrium of Hemoglobin and Its Structural Interpretation. Proc. Natl. Acad. Sci 1935, 21 (4), 186-191.

48. Gesztelyi, R.; Zsuga, J.; Kemeny-Beke, A.; Varga, B.; Juhasz, B.; Tosaki, A., The Hill Equation and the Origin of Quantitative Pharmacology. Archive for History of Exact Sciences 2012, 66 (4), 427-438.

49. Eaton, W. A.; Henry, E. R.; Hofrichter, J.; Mozzarelli, A., Is Cooperative Oxygen Binding by Hemoglobin Really Understood? Nat. Struct. Mol. Biol. 1999, 6(4), 351.

50. Hill, T. L., Theory of Physical Adsorption. In Advances in Catalysis and Related Subjects, Frankenburg, W. G., Ed. Academic Press: New York, 1952; Vol. IV, pp 211-258. 
51. Banerjee, D.; Simon, C. M.; Plonka, A. M.; Motkuri, R. K.; Liu, J.; Chen, X.; Smit, B.; Parise, J. B.; Haranczyk, M.; Thallapally, P. K., Metal-Organic Framework with Optimally Selective Xenon Adsorption and Separation. Nat. Commun. 2016, 7, ncomms11831.

52. Mason, J. A.; Sumida, K.; Herm, Z. R.; Krishna, R.; Long, J. R., Evaluating Metal-Organic Frameworks for Post-Combustion Carbon Dioxide Capture via Temperature Swing Adsorption. Energ. Environ. Sci. 2011, 4 (8), 3030-3040.

53. Hill, T. L., Statistical Mechanics of Adsorption. VI. Localized Unimolecular Adsorption on a Heterogeneous Surface. J. Chem. Phys. 1949, 17(9), 762-771.

54. Honig, J.; Reyerson, L., Adsorption of Nitrogen, Oxygen and Argon on Rutile at Low Temperatures; Applicability of the Concept of Surface Heterogeneity. J. Phys. Chem. 1952, 56 (1), 140-144.

55. Nishihara, H.; Kyotani, T., Zeolite-Templated Carbons - Three-Dimensional Microporous Graphene Frameworks. Chem. Commun. 2018, 54, 5648-5673.

56. Stadie, N. P.; Vajo, J. J.; Cumberland, R. W.; Wilson, A. A.; Ahn, C. C.; Fultz, B., ZeoliteTemplated Carbon Materials for High-Pressure Hydrogen Storage. Langmuir 2012, 28, 1005710063.

57. Nishihara, H.; Fujimoto, H.; Itoi, H.; Nomura, K.; Tanaka, H.; Miyahara, M. T.; Bonnaud, P. A.; Miura, R.; Suzuki, A.; Miyamoto, N., Graphene-Based Ordered Framework with a Diverse Range of Carbon Polygons Formed in Zeolite Nanochannels. Carbon 2018, 129, 854-862.

58. $\quad$ Purewal, J.; Liu, D.; Sudik, A.; Veenstra, M.; Yang, J.; Maurer, S.; Müller, U.; Siegel, D. J., Improved Hydrogen Storage and Thermal Conductivity in High-Density MOF-5 Composites. J. Phys. Chem. C2012, 116(38), 20199-20212.

59. Walton, K. S.; Snurr, R. Q., Applicability of the BET Method for Determining Surface Areas of Microporous Metal-Organic Frameworks. J. Am. Chem. Soc. 2007, 129(27), 8552-8556.

60. Arstad, B.; Fjellvåg, H.; Kongshaug, K. O.; Swang, O.; Blom, R., Amine Functionalised Metal Organic Frameworks (MOFs) as Adsorbents for Carbon Dioxide. Adsorption 2008, 14 (6), 755-762.

61. Dybtsev, D. N.; Chun, H.; Kim, K., Rigid and Flexible: A Highly Porous Metal-Organic

Framework with Unusual Guest-Dependent Dynamic Behavior. Angew. Chem. Int. Ed. 2004, 43 (38), 5033-5036.

62. Geier, S. J.; Mason, J. A.; Bloch, E. D.; Queen, W. L.; Hudson, M. R.; Brown, C. M.; Long, J. R., Selective Adsorption of Ethylene over Ethane and Propylene over Propane in the Metal-Organic Frameworks $\mathrm{M}_{2}$ (dobdc)(M= Mg, Mn, Fe, Co, Ni, Zn). Chem. Sci. 2013, 4(5), 2054-2061.

63. Bao, Z.; Alnemrat, S.; Yu, L.; Vasiliev, I.; Ren, Q.; Lu, X.; Deng, S., Adsorption of Ethane, Ethylene, Propane, and Propylene on a Magnesium-Based Metal-Organic Framework. Langmuir 2011, $27(22), 13554-13562$.

64. Llewellyn, P. L.; Garcia-Rates, M.; Gaberová, L.; Miller, S. R.; Devic, T.; Lavalley, J.-C.; Bourrelly, S.; Bloch, E.; Filinchuk, Y.; Wright, P. A., Structural Origin of Unusual $\mathrm{CO}_{2}$ Adsorption Behavior of a Small-Pore Aluminum Bisphosphonate MOF. J. Phys. Chem. C 2015, 119 (8), 42084216.

65. Simon, C. M.; Braun, E.; Carraro, C.; Smit, B., Statistical Mechanical Model of Gas Adsorption in Porous Crystals with Dynamic Moieties. Proc. Natl. Acad. Sci. 2017, 114 (3), E287-E296.

66. Raschke, M.; Höfer, U., Chemisorption Energy of Hydrogen on Silicon Surfaces. Phys. Rev. $B$ 2001, 63(20), 201303.

67. Rauscher, H., The Interaction of Silanes with Silicon Single Crystal Surfaces: Microscopic Processes and Structures. Surf. Sci. Rep. 2001, 42 (6-8), 207-328.

68. Conner, W. C.; Falconer, J. L., Spillover in Heterogeneous Catalysis. Chem. Rev. 1995, 95 (3), 759-788.

69. Cabria, I.; Lopez, M.; Alonso, J., Shape of the Hydrogen Adsorption Regions of MOF-5 and Its Impact on the Hydrogen Storage Capacity. Phys. Rev. B 2008, 78(20), 205432. 
70. $\quad$ Eddaoudi, M.; Kim, J.; Rosi, N.; Vodak, D.; Wachter, J.; O'Keeffe, M.; Yaghi, O. M., Systematic Design of Pore Size and Functionality in Isoreticular MOFs and Their Application in Methane Storage. Science 2002, 295 (5554), 469-472.

71. Farha, O. K.; Yazaydın, A. Ö.; Eryazici, I.; Malliakas, C. D.; Hauser, B. G.; Kanatzidis, M. G.; Nguyen, S. T.; Snurr, R. Q.; Hupp, J. T., De Novo Synthesis of a Metal-Organic Framework Material Featuring Ultrahigh Surface Area and Gas Storage Capacities. Nat. Chem. 2010, 2 (11), 944.

72. Li, P.; Vermeulen, N. A.; Gong, X.; Malliakas, C. D.; Stoddart, J. F.; Hupp, J. T.; Farha, O. K., Design and Synthesis of a Water-Stable Anionic Uranium-Based Metal-Organic Framework (MOF) with Ultra Large Pores. Angew. Chem. Int. Ed. 2016, 55 (35), 10358-10362.

73. Farha, O. K.; Eryazici, I.; Jeong, N. C.; Hauser, B. G.; Wilmer, C. E.; Sarjeant, A. A.; Snurr, R. Q.; Nguyen, S. T.; Yazaydın, A. O. z. r.; Hupp, J. T., Metal-Organic Framework Materials with Ultrahigh Surface Areas: Is the Sky the Limit? J. Am. Chem. Soc. 2012, 134 (36), 15016-15021.

74. Miranda, R.; Albano, E.; Daiser, S.; Wandelt, K.; Ertl, G., Roughening Transition in Adsorbed Xenon Multilayers. J. Chem. Phys. 1984, 80 (6), 2931-2938.

75. Rouquerol, J.; Llewellyn, P.; Rouquerol, F., Is the BET Equation Applicable to Microporous Adsorbents? Stud. Surf. Sci. Catal. 2007, 160, 49-56.

76. Bae, Y.-S.; Yazaydın, A. O. z. r.; Snurr, R. Q., Evaluation of the BET Method for Determining Surface Areas of MOFs and Zeolites That Contain Ultra-Micropores. Langmuir 2010, 26 (8), 54755483.

77. Gómez-Gualdrón, D. A.; Moghadam, P. Z.; Hupp, J. T.; Farha, O. K.; Snurr, R. Q., Application of Consistency Criteria to Calculate BET Areas of Micro-and Mesoporous Metal-Organic Frameworks. J. Am. Chem. Soc. 2015, 138 (1), 215-224.

78. Sircar, S.; Mohr, R.; Ristic, C.; Rao, M. B., Isosteric Heat of Adsorption: Theory and Experiment. J. Phys. Chem. B1999, 103, 6539-6546.

79. Stadie, N. P. Synthesis and Thermodynamic Studies of Physisorptive Energy Storage Materials. Ph. D. thesis, California Institute of Technology, 2013.

80. Stadie, N. P.; Murialdo, M.; Ahn, C. C.; Fultz, B., Anomalous Isosteric Enthalpy of Adsorption of Methane on Zeolite-Templated Carbon. J. Am. Chem. Soc. 2013, 135(3), 990-993.

81. Shen, D.; Bülow, M.; Siperstein, F.; Engelhard, M.; Myers, A. L., Comparison of Experimental Techniques for Measuring Isosteric Heat of Adsorption. Adsorption 2000, 6 (4), 275-286.

82. Sircar, S., Gibbsian Surface Excess for Gas Adsorption - Revisited. Ind. Eng. Chem. Res. 1999, 38, 3670-3682.

83. Sircar, S., The Genius of Gibbsian Surface Excess (GSE) Framework for Fluid (Gas or Liquid)-Solid Adsorption: A Powerful Practical Tool. Sep. Purif. Technol. 2019, 213, 235-245.

84. Bhatia, S. K.; Myers, A. L., Optimum Conditions for Adsorptive Storage. Langmuir 2006, 22, 1688-1700.

85. Casco, M. E.; Martínez-Escandell, M.; Gadea-Ramos, E.; Kaneko, K.; Silvestre-Albero, J.; Rodriguez-Reinoso, F., High-Pressure Methane Storage in Porous Materials: Are Carbon Materials in the Pole Position? Chem. Mater. 2015, 27(3), 959-964.

86. Kapelewski, M. T.; Runčevski, T. e.; Tarver, J. D.; Jiang, H. Z.; Hurst, K. E.; Parilla, P. A.; Ayala, A.; Gennett, T.; FitzGerald, S. A.; Brown, C. M., Record High Hydrogen Storage Capacity in the MetalOrganic Framework $\mathrm{Ni}_{2}$ (m-dobdc) at near-Ambient Temperatures. Chem. Mater. 2018, 30(22), 8179-8189.

87. Ruthven, D.; Loughlin, K.; Holborow, K., Multicomponent Sorption Equilibrium in Molecular Sieve Zeolites. Chem. Eng. Sci. 1973, 28 (3), 701-709.

88. Schell, J.; Casas, N.; Pini, R.; Mazzotti, M., Pure and Binary Adsorption of $\mathrm{CO}_{2}, \mathrm{H}_{2}$, and $\mathrm{N}_{2}$ on Activated Carbon. Adsorption 2012, 18(1), 49-65.

89. Talu, O., Needs, Status, Techniques and Problems with Binary Gas Adsorption Experiments. Adv. Colloid Interface Sci. 1998, 76-77, 227-269. 
90. Myers, A. L.; Prausnitz, J. M., Thermodynamics of Mixed-Gas Adsorption. AlChE J. 1965, 11 (1), 121-127.

91. Hefti, M.; Marx, D.; Joss, L.; Mazzotti, M., Adsorption Equilibrium of Binary Mixtures of Carbon Dioxide and Nitrogen on Zeolites ZSM-5 and 13X. Micropor. Mesopor. Mater. 2015, 215, 215-228.

92. Schell, J.; Casas, N.; Blom, R.; Spjelkavik, A. I.; Andersen, A.; Cavka, J. H.; Mazzotti, M., MCM41, MOF and UIO-67/MCM-41 Adsorbents for Pre-Combustion $\mathrm{CO}_{2}$ Capture by PSA: Adsorption Equilibria. Adsorption 2012, 18(3-4), 213-227.

93. Yang, Q.; Zhong, C., Molecular Simulation of Carbon Dioxide/Methane/Hydrogen Mixture Adsorption in Metal-Organic Frameworks. J. Phys. Chem. B 2006, 110, 17776-17783.

94. Cessford, N. F.; Seaton, N. A.; Düren, T., Evaluation of Ideal Adsorbed Solution Theory as a Tool for the Design of Metal-Organic Framework Materials. Ind. Eng. Chem. Res. 2012, 51 (13), 4911-4921.

95. Costa, E.; Sotelo, J.; Calleja, G.; Marrón, C., Adsorption of Binary and Ternary Hydrocarbon Gas Mixtures on Activated Carbon: Experimental Determination and Theoretical Prediction of the Ternary Equilibrium Data. AlChE J. 1981, 27 (1), 5-12.

96. Talu, O.; Zwiebel, I., Multicomponent Adsorption Equilibria of Nonideal Mixtures. AlChE J. 1986, 32 (8), 1263-1276.

97. Li, J.-R.; Kuppler, R. J.; Zhou, H.-C., Selective Gas Adsorption and Separation in Metal-Organic Frameworks. Chem. Soc. Rev. 2009, 38, 1477-1504.

98. Hudson, M. R.; Queen, W. L.; Mason, J. A.; Fickel, D. W.; Lobo, R. F.; Brown, C. M., Unconventional, Highly Selective $\mathrm{CO}_{2}$ Adsorption in Zeolite SSZ-13. J. Am. Chem. Soc. 2012, 134 (4), 1970-1973.

99. Li, J.-R.; Ma, Y.; McCarthy, M. C.; Sculley, J.; Yu, J.; Jeong, H.-K.; Balbuena, P. B.; Zhou, H.-C., Carbon Dioxide Capture-Related Gas Adsorption and Separation in Metal-Organic Frameworks. Coord. Chem. Rev. 2011, 255, 1791-1823.

100. Guo, H.; Zhu, G.; Hewitt, I. J.; Qiu, S., "Twin Copper Source” Growth of Metal-Organic Framework Membrane: $\mathrm{Cu}_{3}(\mathrm{BTC})_{2}$ with High Permeability and Selectivity for Recycling $\mathrm{H}_{2}$. J. Am. Chem. Soc. 2009, 131, 1646-1647.

101. Bloch, E. D.; Queen, W. L.; Krishna, R.; Zadrozny, J. M.; Brown, C. M.; Long, J. R., Hydrocarbon Separations in a Metal-Organic Framework with Open Iron (II) Coordination Sites. Science 2012, 335 (6076), 1606-1610.

102. Britt, D.; Tranchemontagne, D.; Yaghi, O. M., Metal-Organic Frameworks with High Capacity and Selectivity for Harmful Gases. Proc. Nat. Acad. Sci. 2014, 105 (33), 11623-11627.

103. Salles, F.; Ghoufi, A.; Maurin, G.; Bell, R. G.; Mellot-Draznieks, C.; Férey, G., Molecular Dynamics Simulations of Breathing MOFs: Structural Transformations of MIL-53 (Cr) Upon Thermal Activation and $\mathrm{CO}_{2}$ Adsorption. Angew. Chem. Int. Ed. 2008, 47(44), 8487-8491. 104. Horike, S.; Shimomura, S.; Kitagawa, S., Soft Porous Crystals. Nat. Chem. 2009, 1 (9), 695. 105. Alhamami, M.; Doan, H.; Cheng, C.-H., A Review on Breathing Behaviors of Metal-OrganicFrameworks (MOFs) for Gas Adsorption. Materials 2014, 7(4), 3198-3250.

106. Krause, S.; Bon, V.; Senkovska, I.; Stoeck, U.; Wallacher, D.; Többens, D. M.; Zander, S.; Pillai, R. S.; Maurin, G.; Coudert, F.-X., A Pressure-Amplifying Framework Material with Negative Gas Adsorption Transitions. Nature 2016, 532 (7599), 348.

107. Evans, J. D.; Bocquet, L.; Coudert, F.-X., Origins of Negative Gas Adsorption. Chem 2016, 1 (6), 873-886.

108. Kitaura, R.; Seki, K.; Akiyama, G.; Kitagawa, S., Porous Coordination-Polymer Crystals with Gated Channels Specific for Supercritical Gases. Angew. Chem. Int. Ed. 2003, 42 (4), 428-431. 109. Mason, J. A.; Oktawiec, J.; Taylor, M. K.; Hudson, M. R.; Rodriguez, J.; Bachman, J. E.; Gonzalez, M. I.; Cervellino, A.; Guagliardi, A.; Brown, C. M., Methane Storage in Flexible Metal-Organic Frameworks with Intrinsic Thermal Management. Nature 2015, 527 (7578), 357-361. 
110. Serre, C.; Bourrelly, S.; Vimont, A.; Ramsahye, N. A.; Maurin, G.; Llewellyn, P. L.; Daturi, M.; Filinchuk, Y.; Leynaud, O.; Barnes, P., An Explanation for the Very Large Breathing Effect of a MetalOrganic Framework During $\mathrm{CO}_{2}$ Adsorption. Adv. Mater. 2007, 19 (17), 2246-2251.

111. Boutin, A.; Coudert, F.-X.; Springuel-Huet, M.-A.; Neimark, A. V.; Férey, G.; Fuchs, A. H., The Behavior of Flexible MIL-53 (Al) Upon $\mathrm{CH}_{4}$ and $\mathrm{CO}_{2}$ Adsorption. J. Phys. Chem. C2010, 114 (50), 22237-22244.

112. Coudert, F.-X.; Jeffroy, M.; Fuchs, A. H.; Boutin, A.; Mellot-Draznieks, C., Thermodynamics of Guest-Induced Structural Transitions in Hybrid Organic-Inorganic Frameworks. J. Am. Chem. Soc. 2008, 130 (43), 14294-14302.

113. Elsaidi, S. K.; Mohamed, M. H.; Simon, C. M.; Braun, E.; Pham, T.; Forrest, K. A.; Xu, W.; Banerjee, D.; Space, B.; Zaworotko, M. J., Effect of Ring Rotation Upon Gas Adsorption in SIFSIX-3-M ( $\mathrm{M}=\mathrm{Fe}, \mathrm{Ni}$ ) Pillared Square Grid Networks. Chem. Sci. 2017, 8(3), 2373-2380.

114. Smit, B.; Maesen, T. L., Molecular Simulations of Zeolites: Adsorption, Diffusion, and Shape Selectivity. Chem. Rev. 2008, 108 (10), 4125-4184.

115. Bering, B.; Dubinin, M.; Serpinsky, V., Theory of Volume Filling for Vapor Adsorption. J. Colloid Interface Sci. 1966, 21 (4), 378-393.

116. Dubinin, M. M., The Potential Theory of Adsorption of Gases and Vapors for Adsorbents with Energetically Nonuniform Surfaces. Chem. Rev. 1960, 60 (2), 235-241.

117. Monsalvo, M. A.; Shapiro, A. A., Study of High-Pressure Adsorption from Supercritical Fluids by the Potential Theory. Fluid Phase Equilib. 2009, 283 (1-2), 56-64.

118. Dundar, E.; Zacharia, R.; Chahine, R.; Bénard, P., Modified Potential Theory for Modeling Supercritical Gas Adsorption. Int. J. Hydrogen Energy 2012, 37, 9137-9147.

119. Henderson, D., Fundamentals of Inhomogeneous Fluids. Marcel Dekker, Inc.: New York 1992.

120. Lastoskie, C.; Gubbins, K. E.; Quirke, N., Pore Size Distribution Analysis of Microporous Carbons: A Density Functional Theory Approach. J. Phys. Chem. 1993, 97(18), 4786-4796.

121. Gelb, L. D.; Gubbins, K.; Radhakrishnan, R.; Sliwinska-Bartkowiak, M., Phase Separation in Confined Systems. Rep. Prog. Phys. 1999, 62 (12), 1573.

122. Rideal, E. K., Dr. Irving Langmuir, For. Mem. R. S. Nature 1957, 180, 581-582.

123. Hull, A. W., Dr. Irving Langmuir's Contributions to Physics. Nature 1958, 181, 148-149.

124. Taylor, H., Irving Langmuir, 1881-1957. Biographical Memoirs of Fellows of the Royal Society 1958, 4, 167-184.

125. Suits, G., The Collected Works of Irving Langmuir. Pergamon Press Ltd.: Oxford, 1961; Vol. 112. 
Table of Contents Graphic

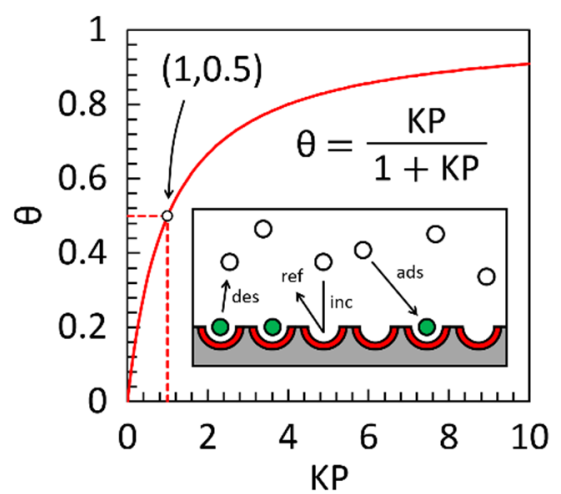




\section{Biographies}

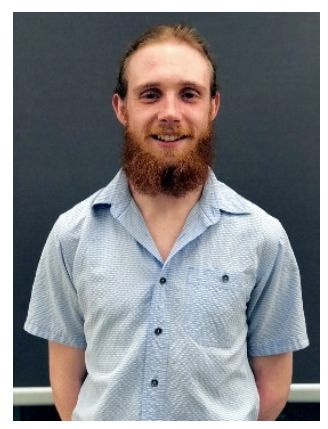

Hans Swenson graduated from high school in Bozeman, Montana, and is currently an undergraduate student at Montana State University, with aspirations of pursuing a $\mathrm{PhD}$ in chemistry. His research has been focused on measuring the adsorption of helium on microporous materials to elucidate the effects of narrow pore size on errors in void volume determination.

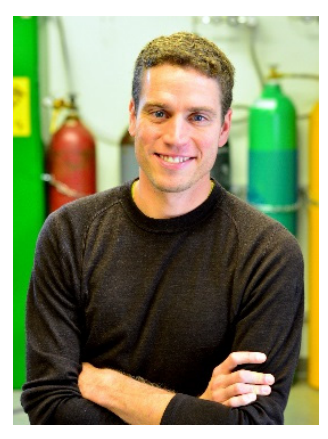

Nicholas P. Stadie received a B.S. in Chemistry from Arizona State University where he performed research on zeolitic imidazolate frameworks (ZIFs) under Prof. Michael O'Keeffe. At the California Institute of Technology, he undertook studies of high surface area carbonaceous framework materials and their gas adsorption properties under Prof. Brent Fultz, receiving a Ph.D. in Materials Science in 2013. His attention turned to reactive, unstable porous phases of hydrogen-rich materials such as magnesium borohydride under Prof. Andreas Züttel at the Swiss Federal Laboratories for Materials Science \& Technology (Empa). After a second postdoctoral position at ETH Zürich applying porous carbon materials as exotic battery electrodes in the laboratory of Prof. Maksym Kovalenko, he returned to North America in 2017 as an assistant professor at Montana State University. His research efforts combine synthetic solid-state chemistry with experimental studies of gas-surface interactions, especially under high-pressure conditions. 